\title{
COMPARATIVE STUDIES OF FISSION FRAGMENT AND ELECTRON BEAM DRIVEN SOLID-STATE LASERS AND REACTOR-LASER SYSTEM MODELING
}

A Dissertation

Presented to

the Faculty of the Graduate School

at the University of Missouri-Columbia

In Partial Fulfillment

of the Requirements for the Degree

Doctor of Philosophy

by

DENIS WISNIEWSKI

Dr. Mark Prelas

DECEMBER 2018 

The undersigned, appointed by the dean of the Graduate School, have examined the dissertation entitled

\section{COMPARATIVE STUDIES OF FISSION FRAGMENT AND ELECTRON BEAM DRIVEN SOLID-STATE LASERS AND REACTOR-LASER SYSTEM MODELING}

Presented by Denis Wisniewski

a candidate for the degree Doctor of Philosophy

and hereby certify that, in their opinion, it is worthy of acceptance.

\begin{tabular}{l}
\hline Professor Mark Prelas \\
\hline Professor Randy Curry \\
\hline
\end{tabular}

Professor Sudarshan Loyalka

Professor Robert Tompson 


\section{Dedications}

In memory of babcia,

Krystina Wisniewski 


\section{Acknowledgements}

I would like to thank my adviser Dr. Prelas for his support and patience. The GAANN fellowship program for their financial support. Behind the scenes support from Latricia Vaughn and James Bennet. And to my family and friends. 


\section{Contents}

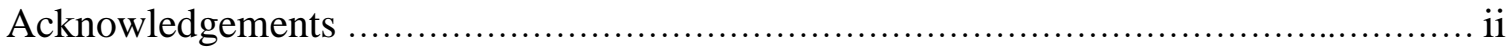

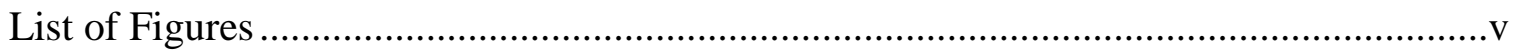

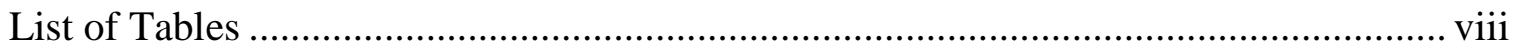

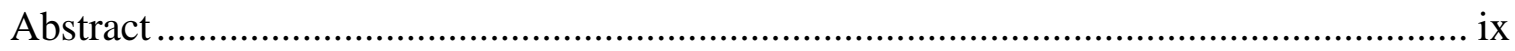

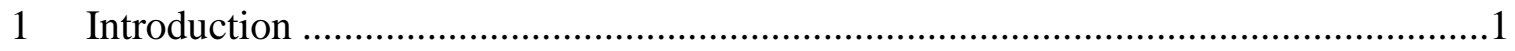

1.1 Solid State Nuclear Pumped Lasers ..........................................................

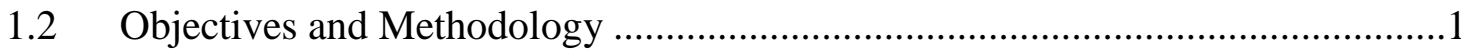

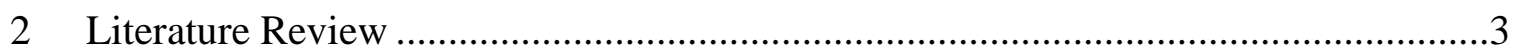

$2.1 \quad$ Review of Nuclear Pumped Lasers ...............................................................

2.2 Review of Electron Beam Pumped Semiconductor Lasers................................6

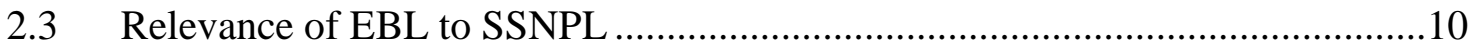

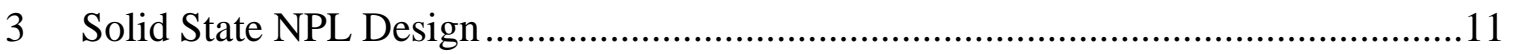

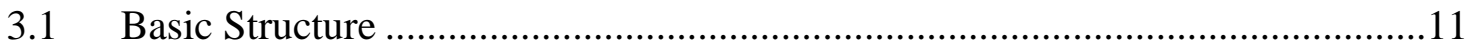

3.2 Spatial Energy Distribution of Fission Fragments: Approximation....................11

3.3 Spatial Energy Distribution of Fission Fragments: MCNP Calculations............14

3.4 MCNP Criticality calculations ..............................................................

3.5 The Role of Defects in Semiconductor Lasers ..............................................19

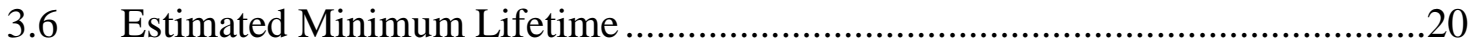


4 Laser Model

4.1 Electron Beam Pumped Semiconductor Laser Model .....................................22

4.2 Constants, Equations, and Pertinent Material Properties .................................22

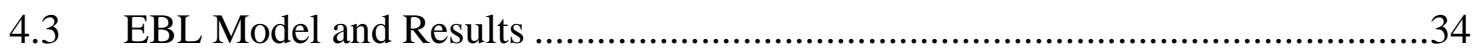

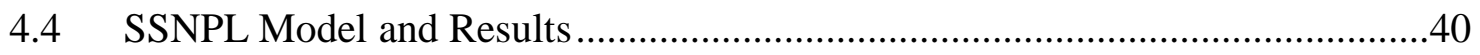

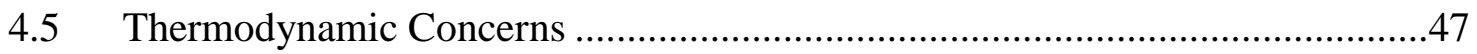

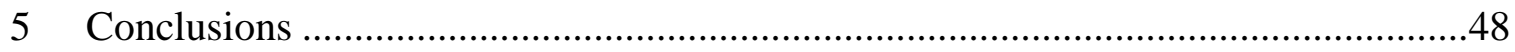

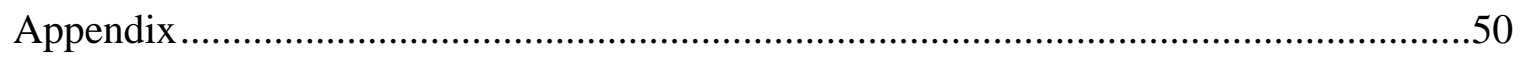

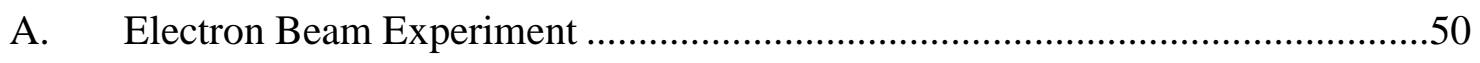

B. Simplification of Permittivity Rate Equation................................................53

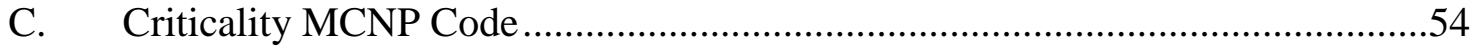

D. Fission Product Transport Code ............................................................55

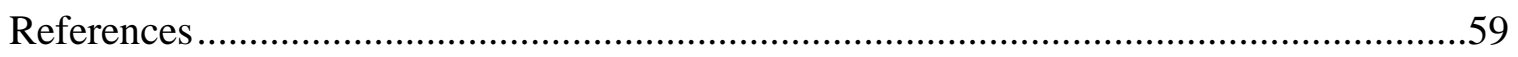

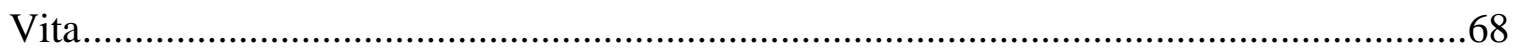




\section{List of Figures}

Figure 1: Comparison of Longitudinal (left) and Transverse (right) electron beam pumped lasers

Figure 2: Staircase geometry. Copper substrate (1) with notched semiconductor material

Figure 3: Single laser module (front view)

Figure 4: Basic model for calculation of $f$. R is the range of a given isotope with its tabulated energy found with SRIM, (a) is the width of the fuel region and $\theta$ is the angle which quantifies what fraction of emitted particles have enough energy to escape the fuel region.

Figure 5: Cut away diagram of modeled MCNP geometry. Grey regions are fuel layer, green region is the semiconductor, and the hatched region is the absorbing carbon sphere. Red circles are example paths fission products could potentially take. Diagram is not to scale

Figure 6: Averaged tally results from TMESH tallies across semiconductor volume. Top left: $3 \mu \mathrm{m}$. Top right: $4 \mu \mathrm{m}$. Bottom left: $5 \mu \mathrm{m}$. Bottom right: $6 \mu \mathrm{m}$. .17

Figure 7: Single cell for criticality calculations. This structure is repeated until it fills the desired volume. (a) is the fuel layer, (b) is the semiconductor layer, and (c) is the diamond layer...

Figure 8: Full 3D Dose results from MCNP simulation. Electron beam impacts from the bottom plane. Units are in $\mathrm{MeV} /\left(\mathrm{cm}^{3}\right.$ source particle $)$.

Figure 9: Plot of raw MCNP data down a center axis perpendicular to the beam axis. The $\mathrm{x}$ values in the legend give the range covered by a particular data cell. 
Figure 10: Plot of uncertainty data down a center axis perpendicular to the beam axis. The $\mathrm{x}$ values in the legend give the range covered by a particular data cell..........................31

Figure 11: Final carrier distribution curve based on MCNP simulations. ........................32

Figure 12: Associated cell uncertainties for MCNP data. During data processing cells with zero dose recorded in them had their uncertainties set to zero. This most likely explains

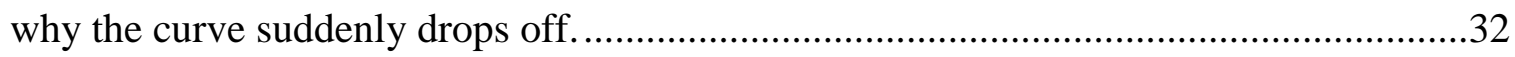

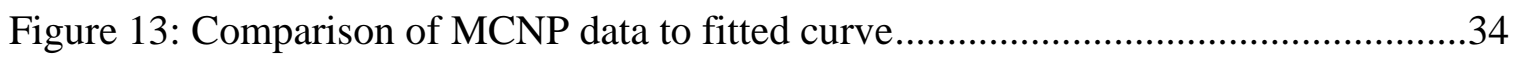

Figure 14: Plot of average laser intensity vs. electron beam current density near threshold.

Figure 15: Full 3D Plot of the laser intensity over space and time. Data taken with $\mathrm{P}=6.36$ $\mathrm{nA} / \mathrm{cm}^{2}$ .38

Figure 16: 1D Plot traced along the peak intensity in figure 15 (left), steady state profile (right). .38

Figure 17: Average laser intensity vs. electron beam current density. The fitted line is given by equation 45 . 39

Figure 18: Steady state solution curves for laser intensity at a given electron beam intensity.

Figure 19: Threshold characteristics for SSNPL for semiconductor thicknesses $3 \mu \mathrm{m}$ (top left), $4 \mu \mathrm{m}$ (top right), $5 \mu \mathrm{m}$ (bottom left), and $6 \mu \mathrm{m}$ (bottom right) .45 Figure 20: Plots of solution taken with $\mathrm{P}=1 \mathrm{MW}$. Steady state profile (left), full time dependent profile (right).

Figure 21: Full experiment setup. (1) The cathode, (2) the emitted beam, (3) the drift tube with gauss coils, (4) CCD camera, (5) Spectrometer fiber input, (6) sample holder..... .52 
Figure 22: CST simulation showing field potential .................................................53

Figure 23: CST simulation showing electron trajectories........................................53 


\section{List of Tables}

Table 1: Most probable isotopes, kinetic energies, and ranges......................................13

Table 2: Fraction of total fission energy deposited into MCNP geometry......................16

Table 3: Summary of critical cubic core properties.................................................19

Table 4: Dislocation estimates for selected heavy ions ...............................................20

Table 5: Evaluated constants and pertinent semiconductor properties ..........................28

Table 6: List of constants and coefficients for SSNPL model. Any constants not listed here are considered to be identical to those from the EBL table.......................................41

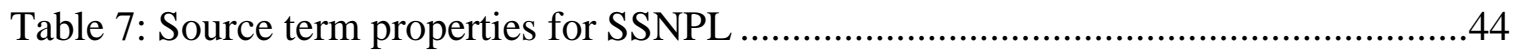

Table 8: Threshold power, power densities, and total efficiency for the laser systems ....46 


\begin{abstract}
A model for a Solid State Nuclear Pumped Laser (SSNPL) system was developed. The model consists of alternating layers of GaN, pure uranium metal, and diamond. The MCNP simulations are used to estimate the minimum core size necessary to achieve criticality. The use of electron beam pumped lasers (EBL) was demonstrated as cost effective analogs for testing SSNPL systems. The reason that EBLs are an effective analog for SSNPL systems is due to a common mechanism responsible for creating the electron-hole pairs in the semiconductor material. A laser model is given and analyzed for a GaAs based EBL system using MCNP simulations of an electron beam. The EBL laser model is modified for fission fragment excitation and applied to the SSNPL system. A system lifetime is estimated based on the dislocations produced by fission products and minimum laser threshold calculations.
\end{abstract}




\section{Introduction}

\subsection{Solid State Nuclear Pumped Lasers}

Lasers pumped by nuclear reactors have the potential to produce high energy/high power beams. The large energy density of nuclear fuels allows for systems significantly smaller those which use traditional power sources. However, due to the nature of fission fragments and their coupling to laser media high power nuclear pumped laser systems are large. Additionally, performing a full scale test for a Nuclear Pumped Laser (NPL) is a long and costly endeavor. The cost of fuel and reactor time severely limits the ability to quickly and easily test potential systems. In this study a Solid State Nuclear Pumped Laser (SSNPL) is developed. It will be demonstrated that a full SSNPL system can reach criticality with a total dimension less than a cubic meter. A cost effective method of testing SSNPLs through Electron Beam Pumped Lasers (EBL) is analyzed.

\subsection{Objectives and Methodology}

A primary design was developed and analyzed. The basic structure of the laser materials and fuel interfaces is given with analysis assuming an optimized geometry based on fission product energy distributions. The effects of defects created by fission products will be quantified based on the various types and their generation rate. The rate at which a semiconductor can repair itself by self-annealing is used to dictate an operating temperature and is discussed. The rate of the defect formation in the laser volume will ultimately dictate the system lifetime.

A laser model will be presented for both an EBL and SSNPL. The EBL model is the far more complicated issue and is discussed first. Data from MCNP simulations will be 
given to demonstrate the true energy deposition profile. This data will then be applied to the EBL model to quantify the results. The SSNPL laser model is closely related to the EBL model, due to commonalities in the mechanisms for the formation of electron-hole pairs, with the major difference being the pumping term. 


\section{Literature Review}

\subsection{Review of Nuclear Pumped Lasers}

Nuclear Pumped Lasers (NPL) are systems where population inversion in a lasing medium is achieved through ionizing radiation from nuclear reactions [1]. NPLs are not a widely studied topic with the majority of research performed by the US in the 70s and 80s. This work was performed largely as part of the Strategic Defense Initiative (SDI) [2]. China [3] and Russia [4-9] have remained more active in the area. The potential high power CW beam produced could be used for missile defense [10], space mining [11], space propulsion [12], power beaming [13], low orbit debris elimination [14], and even meteor deflection [2]. However, the primary driver of research has been military applications.

Nuclear fuels make for an attractive pumping source owing to their immense energy densities. In principle this allows for high energy lasers in small dimensions and reduced external power needs [2]. The major drawback of nuclear fuels are their low power densities, eg the rate at which this energy can be extracted. The fuel materials require neutrons and only reactors can provide the necessary neutron flux. Furthermore, only pulsed reactors can provide a neutron flux large enough to induce lasing [2]. There are only a few ways one could interface the fuel with the lasing media which require low material densities within the system. All NPL systems have been gaseous, except one, which have large lasing power thresholds. With the exception of $\mathrm{CO}_{2}$ and $\mathrm{CO}$, none of the gas mixtures have a particularly high efficiency which further complicates a system's design.

The most common NPLs studied are gaseous where the laser medium was contained within a metal tube. The fuels usually consisted of uranium [15-19] and B-10 [20-22] film 
coatings or He-3 [23-27], $\mathrm{BF}_{3}$ [28], and $\mathrm{UF}_{6}$ [29] gasses mixed with the lasing medium. The gas is kept at around atmospheric pressure giving the nuclear products a range of a few $\mathrm{cm}$. The tubes were designed such that the energy deposited in the gas is nearly uniform down the central axis. Particles born from nuclear reactions are emitted isotopically thus half of all energy from the film coatings fuel is lost to the wall and some of the particles may take paths that do not effectively deposit their energy into the plenum. The range of the particles also puts a constraint on the size of a lasing cavity. If the radius of the cavity were to become too large compared to the average range of a particle very little, if any, energy will be deposited in the center region. Furthermore, the gasses used in previous studies had high power thresholds thus requiring a reactor to be pulsed to produce a large enough neutron flux to reach lasing threshold. In the end such lasers had efficiencies barely above $1 \%$ [2]. The goal of these NPL systems is to group enough tubes together to create a self-critical reactor. To do so with this sort of design leads to large reactor systems [30].

Gaseous fuels counter the primary drawback of film coatings simply because as long as a fuel particle is not near the cavity wall its emitted particles will deposit the entirety of their energy to the surrounding volume. In this case much larger cavity volumes are permitted. The disadvantage of gaseous fuels is there are few to choose from. Fissile fuels must be aerosolized or be contained within another molecule eg. $\mathrm{UF}_{6} . \mathrm{UF}_{6}$ can readily absorb light output from the lasing media and quench lasing action on its own [2]. Theoretical calculations have shown powering an NPL with $\mathrm{UF}_{6}$ is feasible $[31,32]$, and even NASA has made such designs [33], but no NPL has yet to be constructed that does so. It has been suggested that fissile fuel could instead be aerosolized and suspended in the laser cavity [34]. This provides a source of fissile particles without the deleterious effects 
of $\mathrm{UF}_{6}$ such as chemical reactivity and strong light absorption. It should be noted that light absorption from the aerosol will become significant if the particulate density becomes too large. With the fuel being contained in an aerosol particle of finite volume some of the fission energy will be lost to the particle. An exotic fuel type that has been suggested are Uranofullerenes $[35,36]$; uranium atoms trapped within a carbon cage. These could potentially overcome the disadvantages of both other fissile fuel types since fission products need only break out of the carbon cage and it avoids the issue of fluorine chemistry. No designs using Uranofullerenes have been published. The only gaseous fuel that has been used for NPLs is He-3 [23, 25, 37-41]. The energy released from neutron absorption is not large compared to any other fuels discussed but its enormous 5000 barn cross section makes it very efficient with a given neutron flux.

Only one solid state NPL has ever been constructed [41]. This laser was not driven directly by charged particles like other NPL designs. Instead the laser was pumped by a rare gas nuclear driven flash lamp [42] containing He-3. The advantage here is the gas only needs to be induced to fluoresce by a He-3 reaction and then direct that light towards a ND:YAG crystal. Fluorescence requires a significantly lower power density and when this light is concentrated to a much smaller volume it can photolytically pump another medium to lase [43].

The holy grail, so to speak, of NPL would be a self-critical high power steady state (ie. continuous wave) system, which unsurprisingly have been sought after since the first NPLs were demonstrated. This is of particular interest to space based systems [33, 34] where minimization of mass and volume are essential. A self-critical reactor would only require a cooling and reactivity control systems. 
The first NPLs to show lasing were Xe [44] and HF [45] systems pumped by gamma rays from a nuclear weapon detonation. Such lasers were more interest to theory than application since their intent was to prove lasing could be accomplished by ionizing radiation. These systems do however show a close resemblance to Teller's infamous X-ray laser concept [46], where a nuclear warhead is placed within a bundle of Beryllium rods so when detonated the gamma rays from the event will pump the rods and lase before blowing the system apart.

\subsection{Review of Electron Beam Pumped Semiconductor Lasers}

Semiconductor lasers pumped by an electron beam are called Electron Beam Pumped Semiconductor Lasers (referred to as EPL here). Electrons with energies from tens of keV to hundreds of $\mathrm{keV}$ [47] travel through the semiconductor crystal lattice creating electronhole pairs along its path. Unlike injection lasers, e-h pairs must be generated directly from ionizations where that energy is its $W$ value [47] which places the upper limit on the efficiency to any EPL. Many lasers have efficiencies from 1-3\% [48] but some have reported values as high as $10 \%$ [49], 15\% [50], and even 26\% [51]. The lasers are usually analyzed at standard, liquid nitrogen, and liquid helium temperatures. In general it is found power and current thresholds increase with increasing temperatures [52]. The total power output of the lasers can vary widely. Fractions $[48,52]$, tens $[49,53]$, hundreds $[51,54-$ 57], thousands [50, 57-60], and millions [61, 62] of Watts have been reported, however short beam pulses (on the order of $\sim 1 \mathrm{~ns}$ ) put total energy outputs to less than $1 \mathrm{~J}$. CW lasers are difficult to construct due to the power limitations of most electron gun devices operating in steady state conditions. Typical optical power output is on the order of $1 \mathrm{~mW}$ $[52,63]$. Methods to increase effectiveness of $\mathrm{CW}$ lasers include construction of variable 
bandgap structures which reduce the lasing power threshold [64]. It was found materials with larger thermal conductivities have a lower power threshold for a CW state [63].

EPL come in two flavors: longitudinal and transverse. These terms describe whether the optical output leaves the semiconductor parallel to the electron beam (longitudinal) or perpendicular to it (transverse) [Figure 1]. A longitudinal EPL is constructed by taking the sample and coating both sides with a reflective material, the side with the higher reflectivity is pointed towards the e-beam [65]. In some studies the emitting side will not have a reflective coating but will instead have a mirror some distance away from the surface (often called a "radiating mirror") $[59,66-70]$ to reduce beam divergence. Spontaneous emission in transverse directions can negatively affect longitudinal lasers. The effect is usually reduced by cutting small square grooves onto the surface of the semiconductor $[57-59,61,70]$. This hinders the formation of transverse modes by limiting the latitudinal area of the active region.
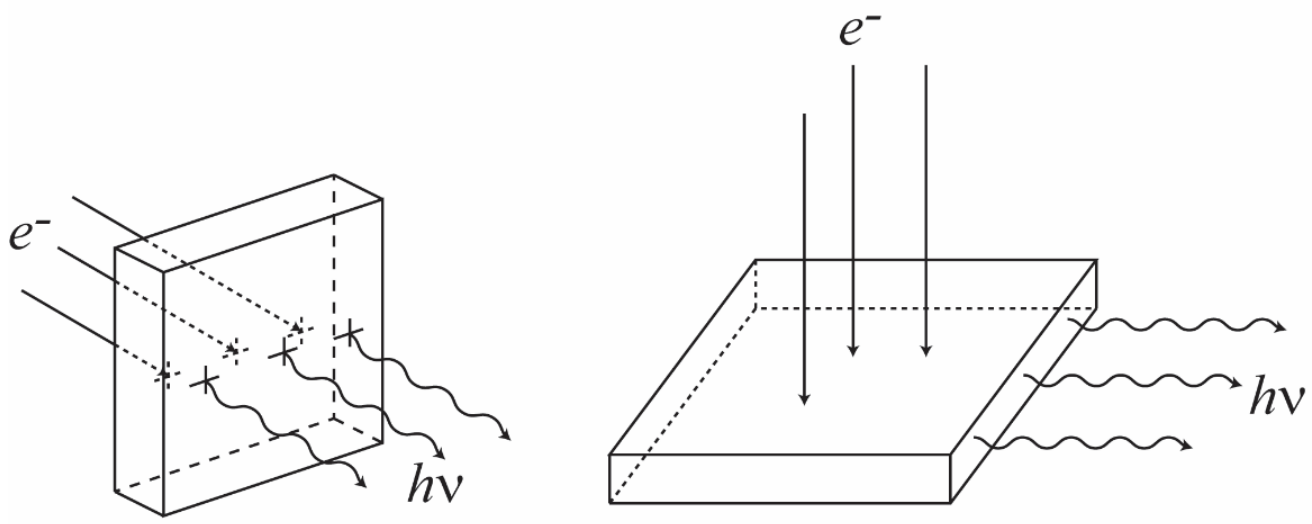

Figure 1: Comparison of Longitudinal (left) and Transverse (right) electron beam pumped lasers 
A transverse EPL has edges of a sample are cleaved along a crystal plane to form resonance cavity with the edges functioning as reflectors [54, 55]. Transverse lasers often suffer more from self-absorption than longitudinal owing to the typically larger volumes needed to be traversed. Some high power transverse lasers were constructed in a stair case geometry with a copper substrate to avoid self absorption [50]. GaAs was attached to each step creating pieces that are small in cross section but long in the perpendicular direction. Light is emitted in the direction perpendicular to the beam and the long axis of the semiconductor [Figure 2]. To prevent transverse modes along the wide portion of the semiconductor grooves were cut along the length analogous to what is done for longitudinally pumped lasers.

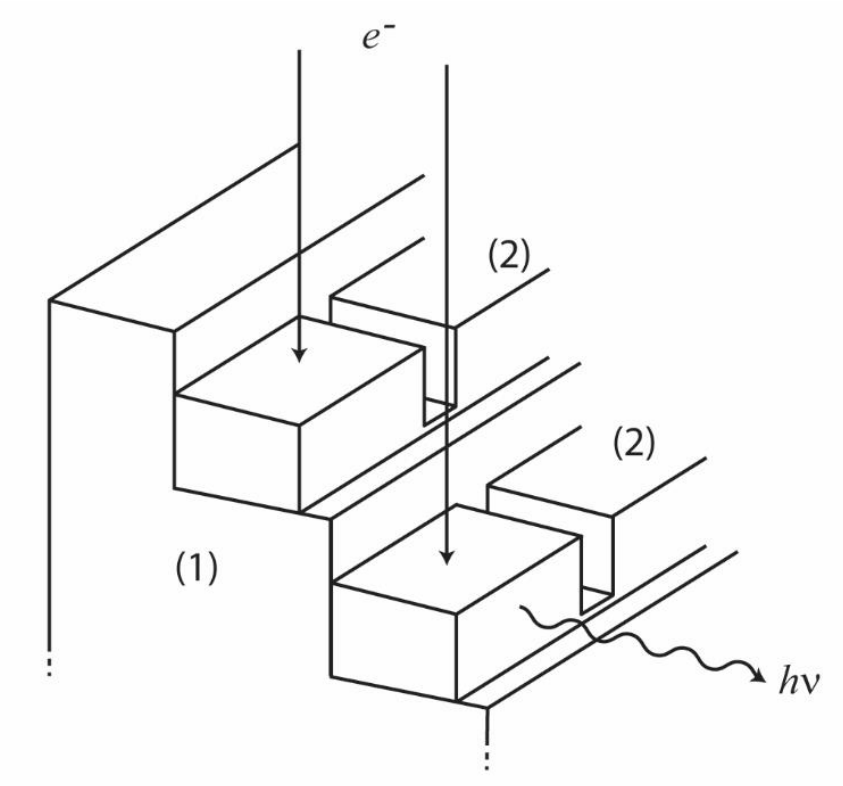

Figure 2: Staircase geometry. Copper substrate (1) with notched semiconductor material (2)

EPL are capable of producing light in a wide spectrum from IR to UV [47]. Particular designs are capable of creating tunable lasers with continuous spectra. The 
technique varies the composition of a semiconductor crystal down a spatial axis $[54,55]$. For example $\mathrm{Zn}_{\mathrm{x}} \mathrm{Cd}_{1-\mathrm{x}} \mathrm{S}$, if $\mathrm{x}$ is continuously changed down an axis of the crystal the bandgap down the axis will vary. The electron beam can then be aimed at any particular spot to produce light with the wavelength equivalent to the bandgap at that position.

The vast majority of research done on EPL has been performed by the Russians [47]. The first to demonstrate lasing from electron irradiation was built by the Russian scientist and Nobel laureate Nikolay Basov [71]. His system used CdS cooled to liquid helium temperatures pumped by $200 \mathrm{keV}$ electrons. It was found that the quality of the crystal is a large factor in the magnitude of threshold power. Basov $[69,72]$ and his coauthor, Bogdankevich [53, 54, 70, 73-77], have been prolific on the subject. Some of the early work on EPL was on the use of self-contained electron beam/semiconductor tubes for television or projectors $[53,72]$, as well as high resolution optical microscopes [75]. Much of the research on EPL through the 80s and 90s centered on binary and tertiary materials, modern research has concentrated on quantum well [78-82] and quantum dot $[78,82]$ heterostructures. Modern research in EBL is virtually nonexistent due to the superior efficiency and simplicity of injection and flash lamp pumped lasers.

The most common materials to be used in EPL are II-VI [48, 66, 72] semiconductors and GaAs $[52,56,67,68,76,83]$. The majority of all studies used doped samples both $\mathrm{p}$ and $\mathrm{n}$ type, various kinds of dopants, and concentrations. The studies on doped and undoped samples show that in general doping always outperforms intrinsic materials [84]. Indirect bandgap semiconductors have yet to produce a laser. It is unclear if indirect is impossible or simply just requires enormous power densities. One paper by Hurwitz [85] showed $\mathrm{GaAs}_{1-\mathrm{x}} \mathrm{P}_{\mathrm{x}}(\mathrm{x}=0.46)$ doped with nitrogen at LN2 temperatures can 
turn an indirect bandgap structure into a quasi-direct structure which achieved lasing. This change was attributed to the presence of nitrogen traps.

\subsection{Relevance of EBL to SSNPL}

EBL and SSNPL are comparable through their mechanisms for achieving population inversion. High energy elections and fission fragments as they pass through matter readily ionize and excite atoms along their paths. While the effective $W$ value (energy expended for e-h production) for heavy ions and electrons are not identical the key species in the creation of e-h pairs are secondary and higher order electrons from collisions. Thus, the net effective $W$ values are equivalent. The spatial distribution of ion pairs, however, are vastly different and will be covered in sections 3.2, 3.3, and 4.4. In this study the semiconductor material used for the EBL is GaAs while in the SSNPL model that material is GaN for its superior properties in harsh environments. 


\section{Solid State NPL Design}

\subsection{Basic Structure}

The primary design of a single laser cell will be alternating layers of nuclear fuel and lasing media. Diamond layers are deposited onto the sides of the cells to serve as neutron moderators and heat conductors. Figure 3 shows a view from the emitting face. The thickness of the fuel (a), semiconductor (b), and diamond (c) can be adjusted as needed for various configurations. The width of the laser/fuel cell is arbitrary and can be set as needed.

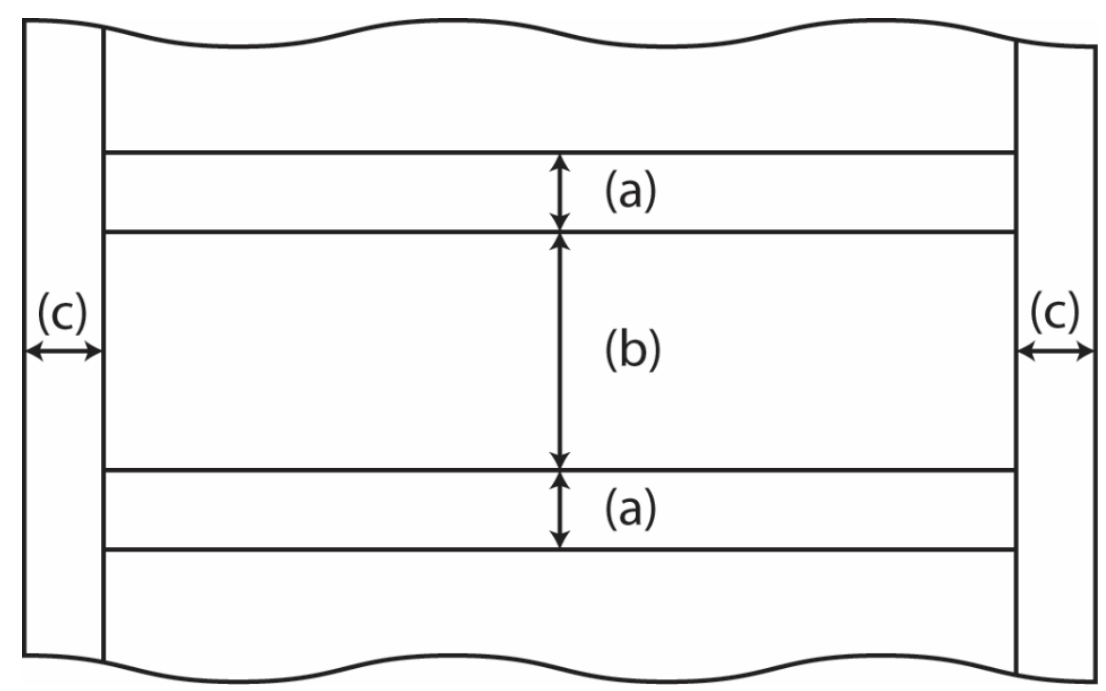

Figure 3: Single laser module (front view)

The dimensions of these cells will be dictated by the range of fission fragments and heat transfer requirements

\subsection{Spatial Energy Distribution of Fission Fragments: Approximation}

The most critical quantity to be determined for the system is the fission fragment range and energy deposition distribution. The range of fission fragments in solid matter is extremely short. The fuel must be thin enough to allow the majority of fission fragments to escape but it cannot be too thin or the power density of the core will be too small. The 
laser cells must be large enough to catch all fission fragments. If the cell is too thin fission fragments could completely traverse the layer and deposit the rest of its energy in the next fuel cell. If the cell is too thick the energy distribution across the cell could drop so low as to hamper, if not inhibit, the ability for the system to lase.

Modeling fission fragments with transport codes to calculate a spatial distribution with transport codes is the most straightforward procedure. The basic assumptions for this model are: The system is critical and in steady state, the fuel is emitting the typical independent thermal neutron induced fission spectrum for U-235. The independent spectrum was chosen because it would most accurately represent the spatial distribution of the actual fission products. A more thorough model would also account for the decay of the fission products. This is beyond the scope of this study.

To a first approximation a heuristic argument to estimate what fraction of energy is deposited in the semiconductor layer is as follows. Consider a fission event in the center of a fuel cell. The isotropic nature of fission dictates that a fragment has equal probability of emission in the spherical solid angle. Given the symmetry of the system considered, this analysis can be confined to two dimensions. The average path length an ion will take in a given material can be calculated based on its stopping power. Given any calculated maximum range for any ion of interest, $R$, it can be calculated what emission angle will result in the particle escaping the fuel layer, see Figure 4. The fraction of ions emitted which penetrate the layer is then based on the angle $\theta$. The total fraction which meets this criteria is then $4 \theta / 2 \pi$. If $L$ is the thickness of the fuel layer this fraction is

$$
f=\frac{2}{\pi} \cos ^{-1}\left(\frac{L}{2 R}\right)
$$


Table 1 is a list of the 20 most common independent fission fragments based on ENDF/B-

VII.1. This table contains the isotope, its mass, its relative probability, its most probable energy, its range in Uranium and GaN, and $f$ calculated for $\mathrm{L}=3,4,5 \mu \mathrm{m}$.

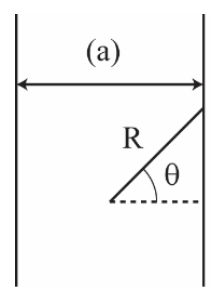

Figure 4: Basic model for calculation of $f$. $R$ is the range of a given isotope with its tabulated energy found with SRIM, (a) is the width of the fuel region and $\theta$ is the angle which quantifies what fraction of emitted particles have enough energy to escape the fuel region.

Table 1: Most probable isotopes, kinetic energies, and ranges

\begin{tabular}{|c|c|c|c|c|c|c|c|c|}
\hline \multirow[t]{2}{*}{ Isotope } & \multirow{2}{*}{$\begin{array}{l}\text { Mass } \\
(\mathrm{amu})^{1}\end{array}$} & \multirow[t]{2}{*}{ Yield $^{2}$} & \multirow{2}{*}{$\begin{array}{l}\text { Kinetic } \\
\text { Energy } \\
(\mathrm{MeV})^{3}\end{array}$} & \multicolumn{2}{|c|}{$\begin{array}{l}\text { Range } \\
(\mu \mathrm{m})^{4}\end{array}$} & \multicolumn{3}{|c|}{$f$} \\
\hline & & & & U & $\mathrm{GaN}$ & $\mathrm{L}=3 \mu \mathrm{m}$ & $\mathrm{L}=4 \mu \mathrm{m}$ & $\mathrm{L}=5 \mu \mathrm{m}$ \\
\hline Te-134 & 133.91154 & $6.22 \mathrm{E}-02$ & 70.10675606 & 4.63 & 8.13 & 0.789962 & 0.71564 & 0.636881 \\
\hline $\mathrm{Zr}-100$ & 99.91776 & $4.98 \mathrm{E}-02$ & 94.19072949 & 5.92 & 9.92 & 0.836916 & 0.780609 & 0.722448 \\
\hline Xe-138 & 137.91399 & $4.81 \mathrm{E}-02$ & 67.68461458 & 4.06 & 7.12 & 0.759087 & 0.672085 & 0.577695 \\
\hline Sr-95 & 94.919358 & $4.54 \mathrm{E}-02$ & 98.02335652 & 5.71 & 9.51 & 0.830776 & 0.772185 & 0.711496 \\
\hline $\mathrm{Kr}-94$ & 93.91536 & $4.51 \mathrm{E}-02$ & 98.73146936 & 5.71 & 9.96 & 0.830776 & 0.772185 & 0.711496 \\
\hline $\mathrm{Kr}-90$ & 89.919524 & $4.40 \mathrm{E}-02$ & 101.5524926 & 5.95 & 9.92 & 0.837757 & 0.781761 & 0.723942 \\
\hline Xe-139 & 138.918787 & $4.32 \mathrm{E}-02$ & 66.97664348 & 4.05 & 7.12 & 0.758462 & 0.671195 & 0.576466 \\
\hline Ba-143 & 142.920617 & 4.10E-02 & 64.15543671 & 4.36 & 7.78 & 0.776411 & 0.696619 & 0.611252 \\
\hline Ba -144 & 143.92294 & $7 \mathrm{E}-02$ & 63.44750738 & 4.34 & 7.76 & 0.775336 & 0.695104 & 609197 \\
\hline Zr-99 & 98.916511 & $3.58 \mathrm{E}-02$ & 95.20135294 & 5.93 & 9.93 & 0.837198 & 0.780994 & 0.722948 \\
\hline Sr-96 & 95.92168 & $3.57 \mathrm{E}-02$ & 97.31538542 & 5.71 & 9.51 & 0.830776 & 0.772185 & 0.711496 \\
\hline Xe-140 & 139.92164 & $3.51 \mathrm{E}-02$ & 66.26853064 & 4.04 & 7.12 & 0.757833 & 0.6703 & 0.575229 \\
\hline $\mathrm{Kr}-89$ & 88.91763 & $3.44 \mathrm{E}-02$ & 101.9894647 & 5.94 & 9.90 & 0.837478 & 0.781378 & 0.723446 \\
\hline Te-135 & 134.91645 & $3.22 \mathrm{E}-02$ & 69.79864706 & 4.63 & 8.19 & 0.789962 & 0.71564 & 0.636881 \\
\hline Xe-137 & 136.907084 & $3.19 \mathrm{E}-02$ & 67.97732775 & 4.05 & 7.12 & 0.758462 & 0.671195 & 0.576466 \\
\hline $\mathrm{Kr}-91$ & 90.92344 & $3.16 \mathrm{E}-02$ & 100.8445633 & 5.96 & 9.92 & 0.838035 & 0.782142 & 0.724437 \\
\hline $\mathrm{Rb}-92$ & 91.919725 & $3.13 \mathrm{E}-02$ & 100.1415751 & 6.08 & 10.0 & 0.841301 & 0.786612 & 0.730231 \\
\hline $\mathrm{Rb}-93$ & 92.922033 & 3.07E-02 & 99.43380404 & 6.08 & 10.1 & 0.841301 & 0.786612 & 0.730231 \\
\hline Ba-142 & 141.916448 & $3.01 \mathrm{E}-02$ & 64.8633533 & 4.37 & 7.8 & 0.776945 & 0.697371 & 0.612272 \\
\hline I-135 & 134.91005 & 2.93E-02 & 69.8039875 & 4.59 & 8.13 & 0.788061 & 0.712981 & 0.633316 \\
\hline
\end{tabular}

${ }^{1}$ Masses from Shultis appendix B [86]. ${ }^{2}$ Yield data from ENDF/B-VII.1[87]. ${ }^{3}$ Kinetic energy based on sister isotopes given a fission emission of 2-3 neutrons. ${ }^{4}$ Ranges calculated with SRIM [88] 
The information shows fuel layers should be no thicker than $5 \mu \mathrm{m}$ while the semiconductor layers should be in the range of 5-10 $\mu \mathrm{m}$. This as it turns out greatly overestimates the ranges of the particles and the energy they deposit along their paths.

\subsection{Spatial Energy Distribution of Fission Fragments: MCNP Calculations}

The modeled MCNP geometry consists of two cylindrical fuel regions sandwiching a semiconductor $(\mathrm{GaN})$ region embedded in a large carbon sphere. See Figure 5 for a cutaway diagram

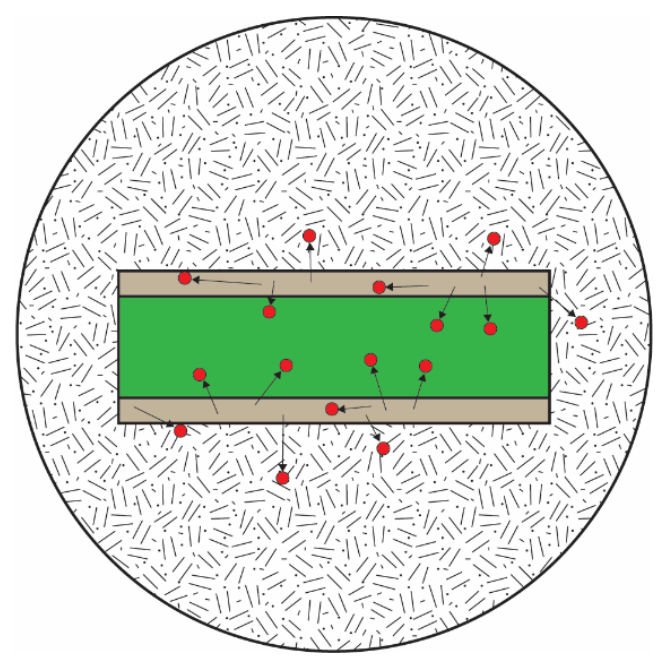

Figure 5: Cut away diagram of modeled MCNP geometry. Grey regions are fuel layer, green region is the semiconductor, and the hatched region is the absorbing carbon sphere. Red circles are example paths fission products could potentially take. Diagram is not to scale

The cylinders have a radius of $0.5 \mathrm{~cm}$ while the sphere has a radius of $10 \mathrm{~cm}$. The radii of the cylinders were chosen to be much wider than their thicknesses such that the energy distribution across the semiconductor layer varies only in the axial direction. The carbon sphere has a much larger radius to ensure all particles including any potential secondary particles are captured. 
There were two sets of tallies calculated in the simulations. First $+\mathrm{F} 6$ tallies were taken in all regions to calculate the total energy deposited in each region from all sources. Second a TMESH tally was taken axially across the semiconductor consisting of 16 evenly spaced mesh points. A representative MCNP code example is given in appendix D.

It was quickly discovered the previous analysis greatly overestimated the optimal thicknesses for the fuel and semiconductor layers. The fuel layers must have a thickness no greater than $1 \mu \mathrm{m}$ and was fixed at this value. The semiconductor regions could have thicknesses no larger than $7 \mu \mathrm{m}$ and it was also found no matter how small this layer was made the energy distribution across the volume was never uniform. The semiconductor layer thicknesses modeled were $3 \mu \mathrm{m}, 4 \mu \mathrm{m}, 5 \mu \mathrm{m}$, and $6 \mu \mathrm{m}$.

The data from the separate simulations can be averaged together through their yield fractions. For a particular region the $+\mathrm{F} 6$ tally could averaged as such

$$
E_{\text {avg }}=\sum_{i} N[\text { particles }] Y_{i} E_{i}\left[\frac{\mathrm{MeV}}{\mathrm{g} \cdot \text { particle }}\right] \rho\left[\frac{\mathrm{g}}{\mathrm{cm}^{3}}\right] V\left[\mathrm{~cm}^{3}\right]
$$

Where $N$ is the number of particles considered, $Y_{i}$ is the yield fraction for a particular isotope, $E_{i}$ is the tally data, $\delta$ is the material density for the region, and $V$ is that region's volume. In this case $N$ would be the number of fissions but in reality the number of fissions is unimportant to this analysis. The quantity of interest is the fraction of energy deposited in each region. Specifically, the energy not deposited into the fuel

$$
f_{n f}=\frac{E_{s}+E_{c}}{\sum_{r} E_{r}}
$$


Where the sum goes over all regions. Considering the true geometry is from Figure 3 it is assumed in the MCNP analysis any energy which would be deposited into the diamond region is deposited into the fuel in the MCNP simulations. It is also assumed any energy deposited by products which travel across both semiconductor and diamond regions is negligible. Table 2 summarizes the relative fractions deposited into each region from the + F6 tallies

Table 2: Fraction of total fission energy deposited into MCNP geometry.

\begin{tabular}{|l|c|c|c|c|c|}
\hline $\begin{array}{c}\text { Semiconductor } \\
\text { Thickness }\end{array}$ & Fuel & Fuel & Semiconductor & $\begin{array}{c}\text { Carbon } \\
\text { Sphere }\end{array}$ & Not in Fuel \\
\hline $3 \mu \mathrm{m}$ & 0.230384 & 0.230262 & 0.332749 & 0.206603 & 0.539352 \\
\hline $4 \mu \mathrm{m}$ & 0.193677 & 0.193558 & 0.302247 & 0.310517 & 0.612764 \\
\hline $5 \mu \mathrm{m}$ & 0.191034 & 0.190908 & 0.308500 & 0.309557 & 0.618057 \\
\hline $6 \mu \mathrm{m}$ & 0.190430 & 0.190302 & 0.309710 & 0.309556 & 0.619266 \\
\hline
\end{tabular}

The Mesh tallies are averaged together in a similar fashion as in equation (2) except mesh tallies are given in units of $\mathrm{MeV} /\left(\mathrm{cm}^{3}\right.$ source particle) so the density is not necessary. Figure 6 shows the axial spatial energy distribution across the semiconductor for the four thicknesses. 

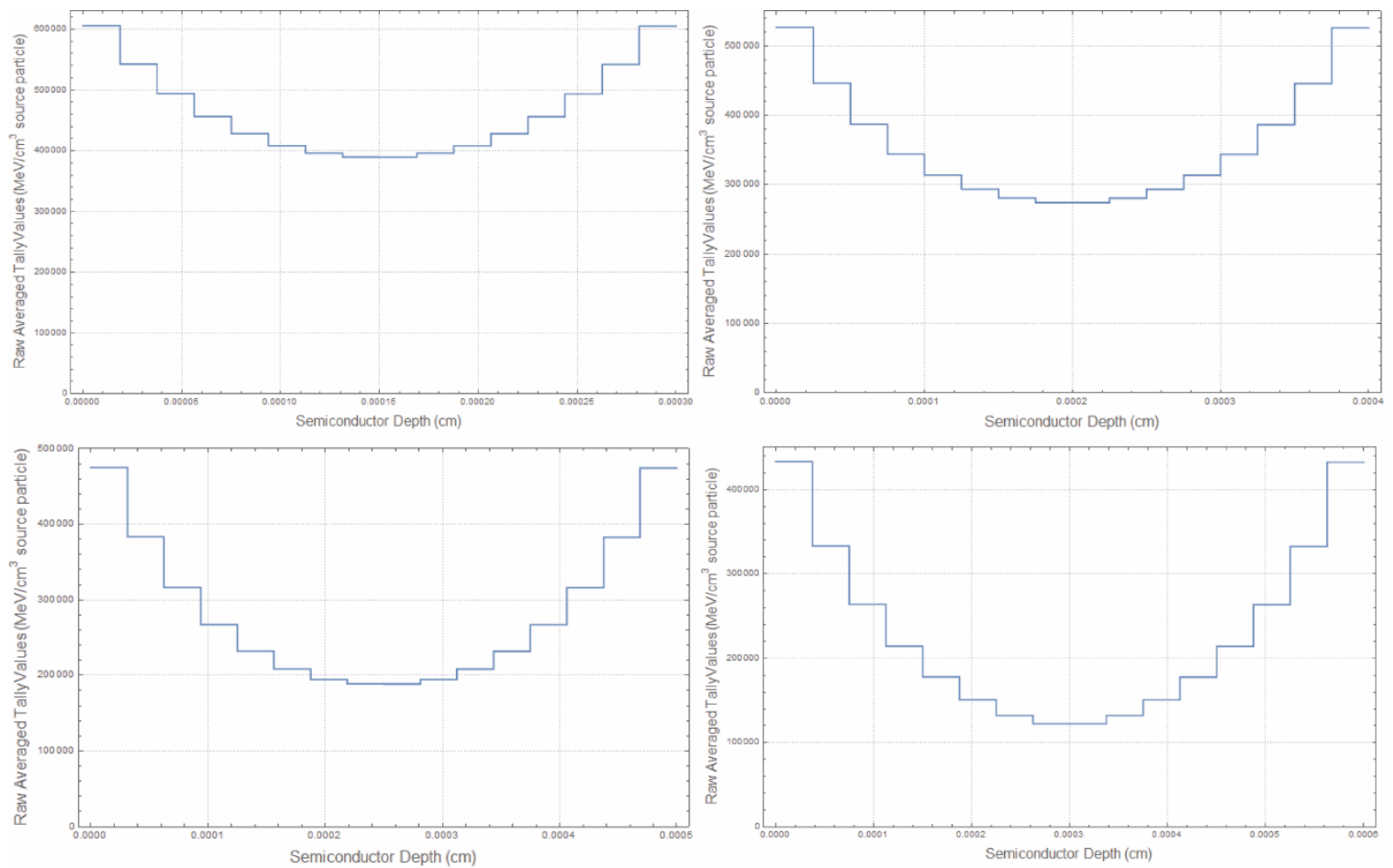

Figure 6: Averaged tally results from TMESH tallies across semiconductor volume. Top left: $3 \mu \mathrm{m}$. Top right: 4 $\mu \mathrm{m}$. Bottom left: $5 \mu \mathrm{m}$. Bottom right: $6 \mu \mathrm{m}$.

\subsection{MCNP Criticality calculations}

A basic criticality calculation can be performed if a few more assumptions are made on the dimensions of the core. First it is assumed the core is cubic, the length of the layers shown in Figure 3 extend the entire volume, the fuel and semiconductor widths are $50 \mu \mathrm{m}$, the fuel is pure U-235 metal, and the diamond layer is $30 \mu \mathrm{m}$ thick. A single cell for computational purposes is given in Figure 7

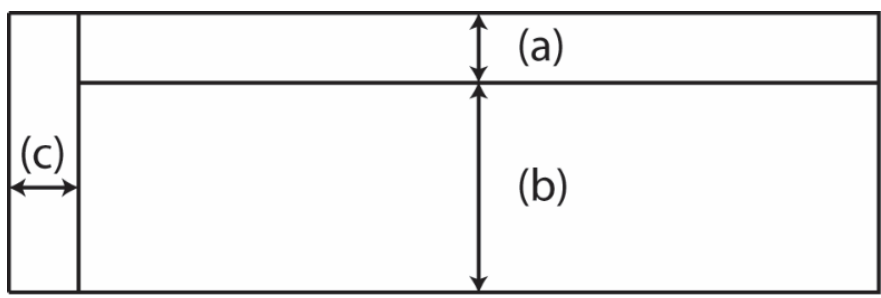

Figure 7: Single cell for criticality calculations. This structure is repeated until it fills the desired volume. (a) is the fuel layer, (b) is the semiconductor layer, and (c) is the diamond layer 
This model allows for a quick and easy calculation of the core density, material ratios, and moderator to fuel ratio before any criticality simulations are performed. The moderator to fuel ratio can be calculated by

$$
\frac{N_{\mathrm{C}}}{N_{\mathrm{U}}}=\frac{A_{\mathrm{C}} L \rho_{\text {diamond }} / m_{\mathrm{C}} \times N_{A}}{A_{\mathrm{U}} L \rho_{\text {uranium }} / m_{\mathrm{U}-235} \times N_{A}}=\frac{A_{\mathrm{C}}}{A_{\mathrm{U}}} \frac{\rho_{\text {diamond }}}{\rho_{\text {uranium }}} \frac{m_{\mathrm{U}-235}}{m_{\mathrm{C}}}
$$

Where $A_{\mathrm{C}}, \rho_{\text {diamond, }}$ and $m_{\mathrm{C}}$ are the face area of the diamond, diamond density, and carbon mass. Likewise $A_{\mathrm{U}}, \rho_{\text {uranium, }}$ and $m_{\mathrm{U}}$ are the face area of the fuel layer, uranium metal density, and U-235 mass.

To perform a criticality calculation the core was be modeled as a homogeneous mass. This can be justified by noting mean free path of a neutron in U-235 is $1 / \Sigma_{t}$, where

$$
\Sigma_{t}=N_{\mathrm{U}} \sigma_{t}=\left(4.89 \times 10^{22} \mathrm{~cm}^{-3}\right)(698.9 \mathrm{~b})=34.18 \mathrm{~cm}^{-1}
$$

Giving a mean free path of approximately $292 \mu \mathrm{m}$, which is much larger than any layer thickness considered. To homogenize the region first consider a cubic core of length $L$ on each side and there are an $N$ number of cells which can fit onto one face. The density of the core is then

$$
\begin{aligned}
\rho_{\text {tot }} & =\frac{V_{U} \rho_{U}+V_{s} \rho_{s}+V_{d} \rho_{d}}{V_{U}+V_{s}+V_{d}}=\frac{N L\left(A_{U} \rho_{U}+A_{s} \rho_{s}+A_{d} \rho_{d}\right)}{N L\left(A_{U}+A_{s}+A_{d}\right)} \\
& =f_{A U} \rho_{u}+f_{A s} \rho_{s}+f_{A d} \rho_{d}
\end{aligned}
$$

Where $f$ is the fractional area each component takes up for one laser cell. 
To calculate minimum criticality the dimensions of the core were varied until a value of $\mathrm{k}>1$ is reached. An example MCNP code can be found in the appendix. Table 3 summarizes the results

Table 3: Summary of critical cubic core properties.

\begin{tabular}{|l|l|l|l|l|}
\hline Thickness & $3 \mu \mathrm{m}$ & $4 \mu \mathrm{m}$ & $5 \mu \mathrm{m}$ & $6 \mu \mathrm{m}$ \\
\hline$f_{A U}$ & 0.15625 & 0.125 & 0.10417 & 0.08929 \\
\hline$f_{A s}$ & 0.46875 & 0.5 & 0.52083 & 0.53571 \\
\hline$f_{A d}$ & 0.375 & 0.375 & 0.375 & 0.375 \\
\hline Core Density (g/cm $\left.{ }^{3}\right)$ & 7.183 & 6.779 & 6.509 & 6.316 \\
\hline MTF Ratio & 8.631 & 10.789 & 12.947 & 15.105 \\
\hline $\mathrm{k}$ & 1.01339 & 1.01915 & 1.00682 & 1.00911 \\
\hline Standard Deviation & 0.00167 & 0.00166 & 0.00165 & 0.00159 \\
\hline Core Length (cm) & 40 & 42 & 45 & 49 \\
\hline Total Core Mass (kg) & 459 & 502 & 593 & 742 \\
\hline
\end{tabular}

Table 3 shows a core of the dimensions specified could easily be launched into space. For reference, the Mars Science Laboratory had a mass of nearly 4 metric tons with a volume of approximately $17 \mathrm{~m}^{3}$ [89].

\subsection{The Role of Defects in Semiconductor Lasers}

As fission products pass through matter in addition to ionizations they will cause a variety of point defects: Voids, dislocations, interstitials, and Frenkel pairs [90, 91]. Large 
scale clusters can form as a result as well. As irradiation continues the various point defects can diffuse to form clusters on their own. Further clustering can form large stable complexes. The presence of these defects create traps for e-h pairs, and scattering/absorption centers for light in the cavity. When the defect density becomes too great lasing action will cease. This is what will ultimately decide the lifetime of a SSNPL. To mitigate this effect the reactor can operate at elevated temperatures high enough to activate annealing. For GaN this range is around $200-400^{\circ} \mathrm{C}$ [92]. Data must be generated to have a clearer picture of repair rates of semiconductors while under irradiation.

\subsection{Estimated Minimum Lifetime}

Even the purest semiconductor materials contain an intrinsic impurity concentration on the order of $10^{16} \mathrm{~cm}^{-3}$. To this end a minimum life time can be estimated by calculating the rate at which impurities are generated in the absence of any annealing effects. Using SRIM the number of dislocations created by a heavy ion are shown in Table 4.

Table 4: Dislocation estimates for selected heavy ions

\begin{tabular}{|l|r|r|}
\hline Heavy lon & Dislocations per ion & Dislocations per MeV \\
\hline $\mathrm{Te}$ & 59000 & 843 \\
\hline $\mathrm{Zr}$ & 39000 & 414 \\
\hline $\mathrm{Xe}$ & 56000 & 823 \\
\hline $\mathrm{Sr}$ & 35000 & 357 \\
\hline $\mathrm{Kr}$ & 34000 & 347 \\
\hline $\mathrm{Ba}$ & 64000 & 1000 \\
\hline $\mathrm{Rb}$ & 34000 & 343 \\
\hline $\mathrm{I}$ & 60000 & 850 \\
\hline
\end{tabular}

Each isotope of the heavy ions have roughly the same kinetic energy and as such all had similar values. In an effort to compress the data into a single parameter to estimate dislocation creation as a whole by the fission process the third column divided the total 
dislocations per ion by its kinetic energy. For a very basic estimate this shows the heavy fission fragments create approximately 800-1000 dislocations per $\mathrm{MeV}$ of kinetic energy, while the light fission fragments create approximately $340-400$ per MeV. The light fission fragments carry roughly two thirds of the fission fragment energy. Thus the total number of dislocations a single fission even can produce in $\mathrm{GaN}$ is

$$
\left[\left(370 \frac{\text { dislocations }}{\mathrm{MeV}}\right) \frac{2}{3}+\left(900 \frac{\text { dislocations }}{\mathrm{MeV}}\right) \frac{1}{3}\right]\left(160 \frac{\mathrm{MeV}}{\text { fission }}\right)=87500 \frac{\text { dislocations }}{\text { fission }}
$$

As discussed in the previous section it is estimated only 50-60\% of the fission energy will make it into the GaN bringing the value of (7) to $43700-52500$ dislocations/fission. The actual value may in fact be lower because the higher dislocation generation rates come from the heavy fragments which have much short ranges, thus depositing a much larger fraction of their energy into the uranium fuel as opposed to the lighter fragments.

If the critical impurity density value for when laser action ceases is set to $10^{18}$ $\mathrm{cm}^{-3}$, the total energy released by fuel can be calculated and a lifetime can be estimated for a given reactor power.

$$
\mathrm{TED}_{\text {max }}=\left(10^{18} \mathrm{~cm}^{-3}\right)\left(160 \frac{\mathrm{MeV}}{\text { fission }}\right)\left(52500 \frac{\text { dislocations }}{\text { fission }}\right)^{-1}=488 \frac{\mathrm{J}}{\mathrm{cm}^{3}}
$$

Where (8) is the cumulative energy density of the core as a whole integrated over its operating lifetime in its most compact state. The laser would be designed with disposability in mind akin to a lightbulb. 


\section{Laser Model}

This section introduces the mathematical model used to analyze both the EBL and SSNPL systems. This model was originally derived with electron beam pumped lasers in mind however by modifying the source term and boundary conditions it can be applied to the SSNPL model. This is due to the fact the key species modeled by the equations is the e-h plasma generated by collisions from electrons/fission products. The EBL model is presented first followed by the SSNPL model.

\subsection{Electron Beam Pumped Semiconductor Laser Model}

Electron beam pumped lasers are able to reach oscillation with pure semiconductor materials. However, a model based on the population difference between electrons and holes is not sufficient. Additionally, due to the nature of free carrier generation through ionization the distribution of e-h pairs is not uniform across the volume. By considering a temporally and spatially dependent complex permittivity coefficient these issues can be accounted for. This is the approach Bogdankevich and others have taken to solve these problems [93-96].

\subsection{Constants, Equations, and Pertinent Material Properties}

The semiconductor is assumed to have a spatially and temporally varying relative permittivity coefficient of the form

$$
\varepsilon(x, t)=\varepsilon_{r}+\delta \varepsilon(x, t)+i\left(\varepsilon_{0}^{\prime \prime}-\varepsilon^{\prime \prime}(x, t)\right)
$$

Where $\varepsilon_{r}$ is the relative permittivity in the absence of any field, $\delta \varepsilon$ represents the change in refractive index due to the inhomogeneous e-h plasma, $\varepsilon_{0}^{\prime \prime}$ relates the permittivity to the 
photon lifetime $\left(\tau_{p}\right)$ and $\varepsilon^{\prime \prime}$ relates the electron population which contributes to gain. In real lasers $\delta \varepsilon, \varepsilon^{\prime \prime}$, and $\varepsilon_{0}^{\prime \prime}$ are typically on the order of $10^{-6}-10^{-4}$. These terms are defined as follows

$$
\begin{gathered}
\varepsilon^{\prime \prime}(x, t)=\frac{\sigma}{k} N(x, t) \\
\varepsilon_{0}^{\prime \prime}=\frac{1}{\omega \tau_{p}}, \tau_{p}=\frac{2 L}{c}\left(\ln \frac{1}{R_{1} R_{2}}+2 \alpha L_{c}\right)^{-1} \\
\delta \varepsilon(x, t)=-\frac{4 \pi e^{2}}{m^{*} \varepsilon_{0} \omega^{2}} N(x, t)=-\frac{4 \pi e^{2}}{m^{*} \varepsilon_{0} \omega^{2}} \frac{k}{\sigma} \varepsilon^{\prime \prime}(x, t)=-\chi_{1} \varepsilon^{\prime \prime}(x, t)
\end{gathered}
$$

Where $\omega$ is the angular frequency of the principle laser emission line, $L$ is the cavity length, $R_{1}$ and $R_{2}$ are the reflectivities of the front and back mirrors, $\alpha$ is the linear loss coefficient, $e$ is the electron charge, $m^{*}$ is the electron effective mass, $\varepsilon_{0}$ is the vacuum permittivity constant, $k$ is the wave vector, and $\sigma$ is the emission cross section. Specifically, in the literature $\sigma$ was defined as "the cross section for radiative recombination averaged over the linewidth. [93]" In this study the definition used for $\sigma$ is

$$
\sigma=\langle\sigma(v)\rangle=\frac{1}{\Delta v} \int_{v_{0}-\Delta v / 2}^{v_{0}+\Delta v / 2} \frac{c^{2}}{8 \pi v^{2} \tau_{1}} g_{v}(v) d v
$$

Where $v_{0}$ is the centerline frequency, $\tau_{1}$ is the free carrier lifetime, $c$ is the speed of light, $g_{\imath}(v)$ is the spectrum lineshape, and $\Delta v$ is the spectrum line width. This is essentially the average integral of the typical stimulated emission cross section found in many laser texts [97-99]. It was chosen to use this form to keep in line with the literature. 
The line width is dictated by various forms of broadening mechanisms. Each broadening mechanism will have an associated lineshape function: Lorentzian for homogeneous broadening, and Gaussian for inhomogeneous broadening. The two considered in this study are natural broadening (homogeneous) and temperature broadening (inhomogeneous).

Natural broadening is related to the difference in lifetimes of upper and lower lasing states. For a generic laser system it is given as

$$
\Delta v_{N}=\frac{1}{2 \pi}\left(\frac{1}{\tau_{1}}+\frac{1}{\tau_{2}}\right)
$$

In the case of a semiconductor laser system the lower state is stable and hence one of the terms in the parentheses becomes zero. Its associated lineshape is a Lorentzian given by

$$
g_{v}(v)=\frac{\left(\Delta v_{N} / 2 \pi\right)}{\left(v-v_{0}\right)^{2}+\left(\Delta v_{N} / 2\right)^{2}}
$$

Temperature broadening (also known as Doppler broadening) is due to the random motion of atoms within the lasing medium. At high temperatures this can be a significant effect and will be of importance to this study. Temperature broadening and Gaussian lineshapes are defined [100] as

$$
\begin{gathered}
\Delta v_{T}=2 v_{0} \sqrt{\frac{2 k_{B} T \ln 2}{M c^{2}}} \\
g_{v}(v)=\frac{2 \sqrt{\ln 2}}{\pi \Delta v_{T}} \exp \left[-4 \ln 2 \frac{\left(v-v_{0}\right)^{2}}{\Delta v_{T}^{2}}\right]
\end{gathered}
$$


Where $k_{B}$ is Boltzmann's constant, $M$ is the mass of the colliding species, and $T$ is the temperature of the medium. For this study only binary semiconductor materials (semiconductor materials made from two different atoms) are considered and $M$ will simply be the average of the atomic masses. Equation (13) can be explicitly be written for both forms of broadening in a simplified form. If the dimensionless variable $v=v o q$ is introduced the Lorentzian cross section becomes

$$
\sigma_{L}=\frac{1}{\tau_{1} v_{0}^{3}}\left(\frac{c}{4 \pi}\right)^{2} \int_{1-\frac{\Delta v}{v_{0}} \frac{\Delta}{2}}^{1+\frac{\Delta v 1}{v_{0}} \frac{1}{2}} q^{-2}\left((q-1)^{2}+\left(\frac{\Delta v}{v_{0}} \frac{1}{2}\right)^{2}\right)^{-1} d q
$$

A similar expression is found for a Gaussian cross section where a new term is defined, $\Delta v_{T}=v_{0} q_{0}$

$$
\sigma_{G}=\frac{2 \sqrt{\ln 2}}{8 \pi^{2}}\left(\frac{c}{v_{0} q_{0}}\right)^{2} \frac{1}{v_{0} \tau_{1}} \int_{1-q_{0} / 2}^{1+q_{0} / 2} q^{-2} \exp \left(-4 \ln 2 \frac{(q-1)^{2}}{q_{0}^{2}}\right) d q
$$

To model the dynamics of a system like an EBL, the basic rate equations are not sufficient because the spatial distribution of active atoms in most lasers is irrelevant by design. This is not the case for electron beam pumped lasers where the nature of the interactions between high energy electrons and matter produce an inhomogeneous dose region, and hence, creates an inhomogeneous e-h plasma. To model the laser intensity as a function of space one must appeal to the fundamental equations for electromagnetic fields. Assuming a nonmagnetic material $(\mu=1)$, the electric field in the cavity is

$$
\nabla^{2} \mathscr{E}=\frac{1}{c^{2}} \frac{\partial^{2}}{\partial t^{2}}(\varepsilon \mathscr{E})
$$


It is assumed the derivatives of the permittivity are small compared to those of the field [95] and so $\varepsilon$ may be pulled out of the time derivatives. Next, if it is assumed the spatial variance is only in the $\mathrm{x}$-direction and thus the electric field is assumed to have the form $\mathcal{E}=E(x, t) \exp [i(\omega t-k z)][96]$ and so equation $(20)$ becomes

$$
\frac{\partial^{2} E}{\partial x^{2}}-k^{2} E=\frac{\varepsilon}{c^{2}}\left(\frac{\partial^{2} E}{\partial t^{2}}-2 i \omega \frac{\partial E}{\partial t}-\omega^{2} E\right)
$$

It is also assumed the laser frequency is much greater than the time derivatives of the field, thus the second order time derivative of the field can be ignored. Applying the definition of $\varepsilon$ in equation (9) and stating $k^{2}=\omega^{2} \varepsilon_{r} / c^{2}$ the equation for the field can be derived

$$
\begin{gathered}
\frac{\partial^{2} E}{\partial x^{2}}-\frac{\varepsilon_{r} \omega^{2}}{c^{2}} E=\frac{\varepsilon_{r}+\delta \varepsilon+i\left(\varepsilon_{0}^{\prime \prime}-\varepsilon^{\prime \prime}\right)}{c^{2}}\left(-2 i \omega \frac{\partial E}{\partial t}-\omega^{2} E\right) \\
\frac{c^{2}}{\varepsilon_{r}} \frac{\partial^{2} E}{\partial x^{2}}-\omega^{2} E=\frac{\varepsilon_{r}+\delta \varepsilon+i\left(\varepsilon_{0}^{\prime \prime}-\varepsilon^{\prime \prime}\right)}{\varepsilon_{r}}\left(-2 i \omega \frac{\partial E}{\partial t}\right)-\omega^{2} E-\frac{\delta \varepsilon+i\left(\varepsilon_{0}^{\prime \prime}-\varepsilon^{\prime \prime}\right)}{\varepsilon_{r}} \omega^{2} E \\
\omega^{2}\left(\frac{c^{2}}{\varepsilon_{r} \omega^{2}} \frac{\partial^{2} E}{\partial x^{2}}+\frac{\delta \varepsilon+i\left(\varepsilon_{0}^{\prime \prime}-\varepsilon^{\prime \prime}\right)}{\varepsilon_{r}} E\right)=-2 i \omega \frac{\varepsilon_{r}+\delta \varepsilon+i\left(\varepsilon_{0}^{\prime \prime}-\varepsilon^{\prime \prime}\right)}{\varepsilon_{r}} \frac{\partial E}{\partial t} \\
\frac{\omega}{2}\left(\frac{i}{k^{2}} \frac{\partial^{2} E}{\partial x^{2}}+\frac{\varepsilon^{\prime \prime}+i \delta \varepsilon-\varepsilon_{0}^{\prime \prime}}{\varepsilon_{r}} E\right)=\frac{\partial E}{\partial t}
\end{gathered}
$$

Where in the final step $\varepsilon / \varepsilon_{r} \sim 1$ with $\varepsilon_{r} \gg>\delta, \varepsilon_{0}^{\prime \prime}, \varepsilon^{\prime \prime}$.

The rate equation for the permittivity was given [93] as

$$
\frac{\partial \varepsilon^{\prime \prime}}{\partial t}+\frac{1}{\tau_{1}} \varepsilon^{\prime \prime}=\frac{\sigma}{k} g(x)-2 \sigma I \varepsilon^{\prime \prime}
$$


Where $g(x)$ is the spatially dependent carrier generation rate. $I$ is the field intensity given by

$$
I(x, t)=\frac{1}{\hbar \omega} \frac{c}{2} \varepsilon_{0}|E(x, t)|^{2}=\chi_{0}|E(x, t)|^{2}\left[\frac{\text { photons }}{\mathrm{cm}^{2} \cdot \mathrm{s}}\right]
$$

Equations (23) and (24) are reduced to nondimensionalized forms by defining the variables: $x=\xi / k, t=\tau_{1} S$

$$
\begin{aligned}
\frac{2}{\omega \tau_{1}} \frac{\partial U}{\partial s} & =i \frac{\partial^{2} U}{\partial \xi^{2}}+\frac{\left(1-i \chi_{1}\right) \varepsilon^{\prime \prime}-\varepsilon_{0}^{\prime \prime}}{\varepsilon_{r}} U \\
\frac{\partial \varepsilon^{\prime \prime}}{\partial s} & =Q(\xi)-\left(1+\delta^{2}|U|^{2}\right) \varepsilon^{\prime \prime}
\end{aligned}
$$

Where the new terms are defined as

$$
E(x, t) \rightarrow u_{0} U(\xi, s), Q(\xi)=\tau_{1} \frac{\sigma}{k} g(\xi), \delta^{2}=2 \tau_{1} \sigma \chi_{0} u_{0}^{2}
$$

Where $u_{0}$ is a scaling constant which carriers the dimensions of the field. Its value is arbitrary and is set to $1 \mathrm{~V} / \mathrm{m}$.

The next step is to determine the carrier generation distribution. Previous work assumed the distribution took the form of a Gaussian [93] and $\operatorname{Sech}^{2}(x)[94,96]$. The Gaussian distribution is the more accurate representation, however, the $\mathrm{Sech}^{2}$ model permits exact solutions in the form of hypergeometric functions. This requires the additional assumption $\varepsilon^{\prime \prime}(x, t) \sim g(x)$, which is equivalent to a zeroth order solution to (27) (see appendix B). To calculate the number of carriers generated an MCNP simulation 
will calculate energy deposition as a function of volume. The carrier number is calculated by dividing the energy values by the $W$ value of the material, which is approximated by the Klein formula [101]

$$
W=2.8 \times E_{g}+0.5[\mathrm{eV}]
$$

The following table gives a list of constants and terms evaluated for GaAs.

Table 5: Evaluated constants and pertinent semiconductor properties

\begin{tabular}{|c|c|c|c|}
\hline Name & Symbol & Value & Units \\
\hline Free Carrier Lifetime & $\tau_{1}$ & $\sim 10^{-8}$ & $\mathrm{~S}$ \\
\hline Photon Frequency $(1.42 \mathrm{eV})$ & $v_{0}$ & $3.43 \times 10^{14}$ & $\mathrm{~s}^{-1}$ \\
\hline$W$ Value $(29)$ & $W$ & 4.4 & $\mathrm{eV}$ \\
\hline Cavity Length & $L$ & 1 & $\mathrm{~cm}$ \\
\hline GaAs Index of Refraction & $n$ & 3.6 & unitless \\
\hline Relative Permittivity Constant & $\varepsilon_{r}$ & 12.9 & unitless \\
\hline Photon Lifetime (11) & $\tau_{p}$ & $2.07 \times 10^{-10}$ & $\mathrm{~s}$ \\
\hline Threshold Permittivity Constant (11) & $\varepsilon 0^{\prime \prime}$ & $2.4 \times 10^{-6}$ & unitless \\
\hline Speed of Light & $c$ & $3 \times 10^{10}$ & $\mathrm{~cm} / \mathrm{s}$ \\
\hline Front Plane Reflectivity & $R$ & 0.32 & unitless \\
\hline Natural Broadening & $\Delta v_{N}$ & $1.95 \times 10^{7}$ & $\mathrm{~s}^{-1}$ \\
\hline SE Cross Section, Lorentzian (18) & $\sigma_{L}$ & $9.549 \times 10^{-10}$ & $\mathrm{~cm}^{2}$ \\
\hline Electron Effective mass & $m *$ & $0.063 m_{e}$ & $\mathrm{MeV} / \mathrm{c}^{2}$ \\
\hline Intensity Coefficient (25) & $\chi_{0}$ & $5.838 \times 10^{11}$ & $\mathrm{~s}^{-1} \mathrm{~V}^{-2}$ \\
\hline
\end{tabular}




\begin{tabular}{|l|l|l|l|}
\hline Second Relative Permittivity Coefficient (12) & $\chi_{1}$ & $2.973 \times 10^{-5}$ & unitless \\
\hline Delta coefficient (28) & $\delta$ & $3.608 \times 10^{-3}$ & unitless \\
\hline
\end{tabular}

In MCNP a $1 \mathrm{~cm}$ diameter electron beam with electron energy E=100 keV was modeled impacting a slab of GaAs. The slab was a $1 \mathrm{~cm} \times 1 \mathrm{~cm} \times 0.06 \mathrm{~cm}$ block divided into a $100 \times 100 \times 100$ mesh. A total of 500 million electrons were transported averaged across 10 simulations of 50 million apiece. Each simulation used the L' Ecuyer pseudorandom number generator rather than the standard MCNP default because of its significantly larger period $\left(9.2 \times 10^{19}\right.$ vs $\left.7.0 \times 10^{13}\right)$. Each simulation had its own unique seed number and number starting position to ensure no crossover from the random number generators. In total the simulations had a run time of approximately three weeks. If the simulations are in fact unique the data from each result can be averaged together. The uncertainties for each cell are averaged in accordance with typical independent measurements [102]

$$
\bar{x}=\frac{1}{N} \sum_{l=1}^{N} x_{l}, \bar{\sigma}=\left(\sum_{l=1}^{N} \frac{1}{\sigma_{l}^{2}}\right)^{-1 / 2}
$$

Where $\bar{x}$ and $\bar{\sigma}$ are the averages for a single cell over the $l$ simulations. A full 3D plot of the raw data is given in Figure 8 


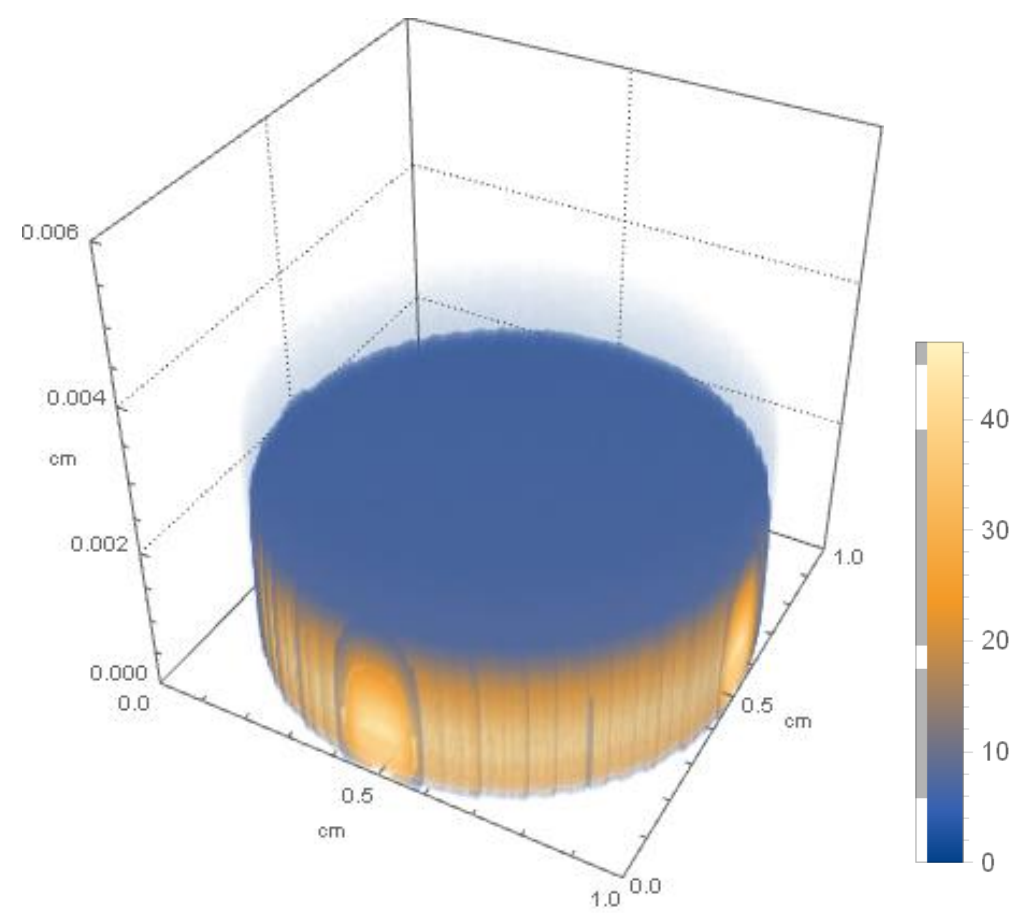

Figure 8: Full 3D Dose results from MCNP simulation. Electron beam impacts from the bottom plane. Units are in $\mathrm{MeV} /\left(\mathrm{cm}^{3}\right.$ source particle)

To simplify the model such that only one spatial axis is considered, the center row down one axis perpendicular to the beam was used. The data is symmetric in the plane so which axis is chosen is irrelevant. Examination of the data along this row shows the data values and uncertainties are approximately constant, and as such the values are averaged over the volume. In general it is not at all accurate to average uncertainties like other normal quantities, however, the argument presented here is this represents the average of the uncertainty over the volume and not the average of various uncertainties from separate measurements. 


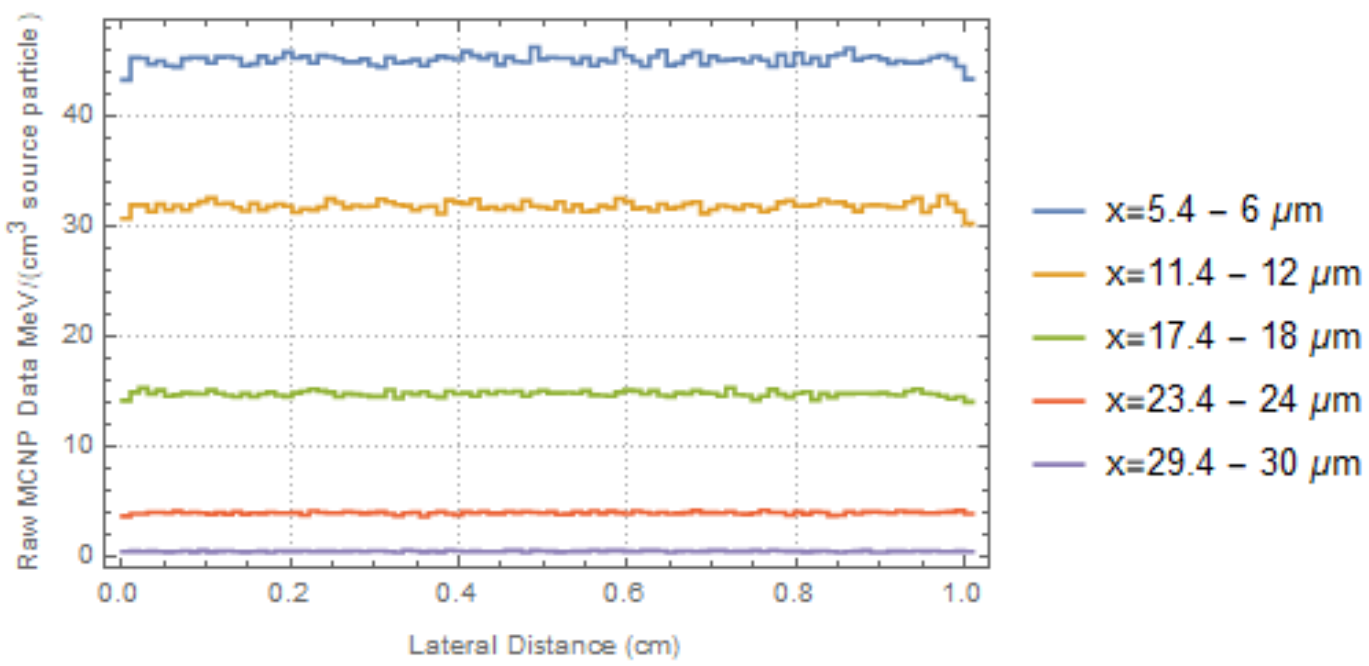

Figure 9: Plot of raw MCNP data down a center axis perpendicular to the beam axis. The $x$ values in the legend give the range covered by a particular data cell.

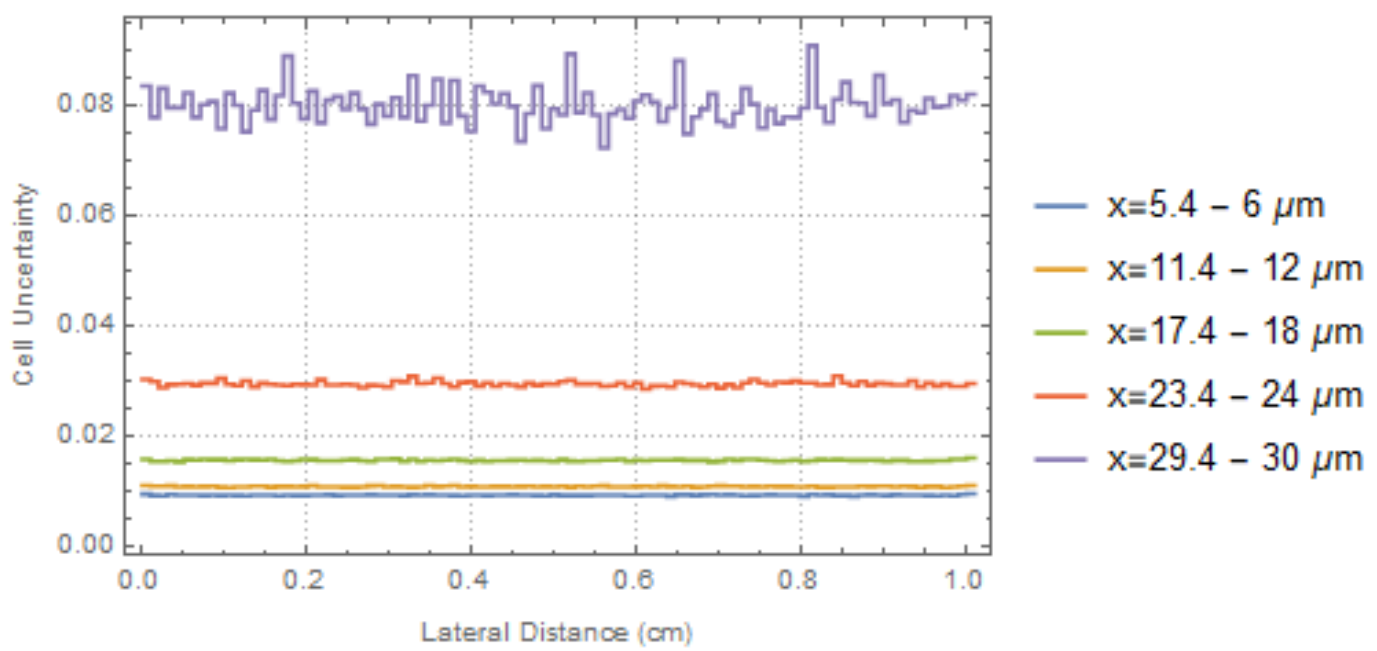

Figure 10: Plot of uncertainty data down a center axis perpendicular to the beam axis. The $x$ values in the legend give the range covered by a particular data cell.

With these assumptions the final laser geometry considered is a $1 \mathrm{~cm} \times 100 \mu \mathrm{m} \times 60 \mu \mathrm{m}$ crystal. Down this axis the final MCNP data is shown in Figure 11 where Figure 12 is a plot of the uncertainties at each step 


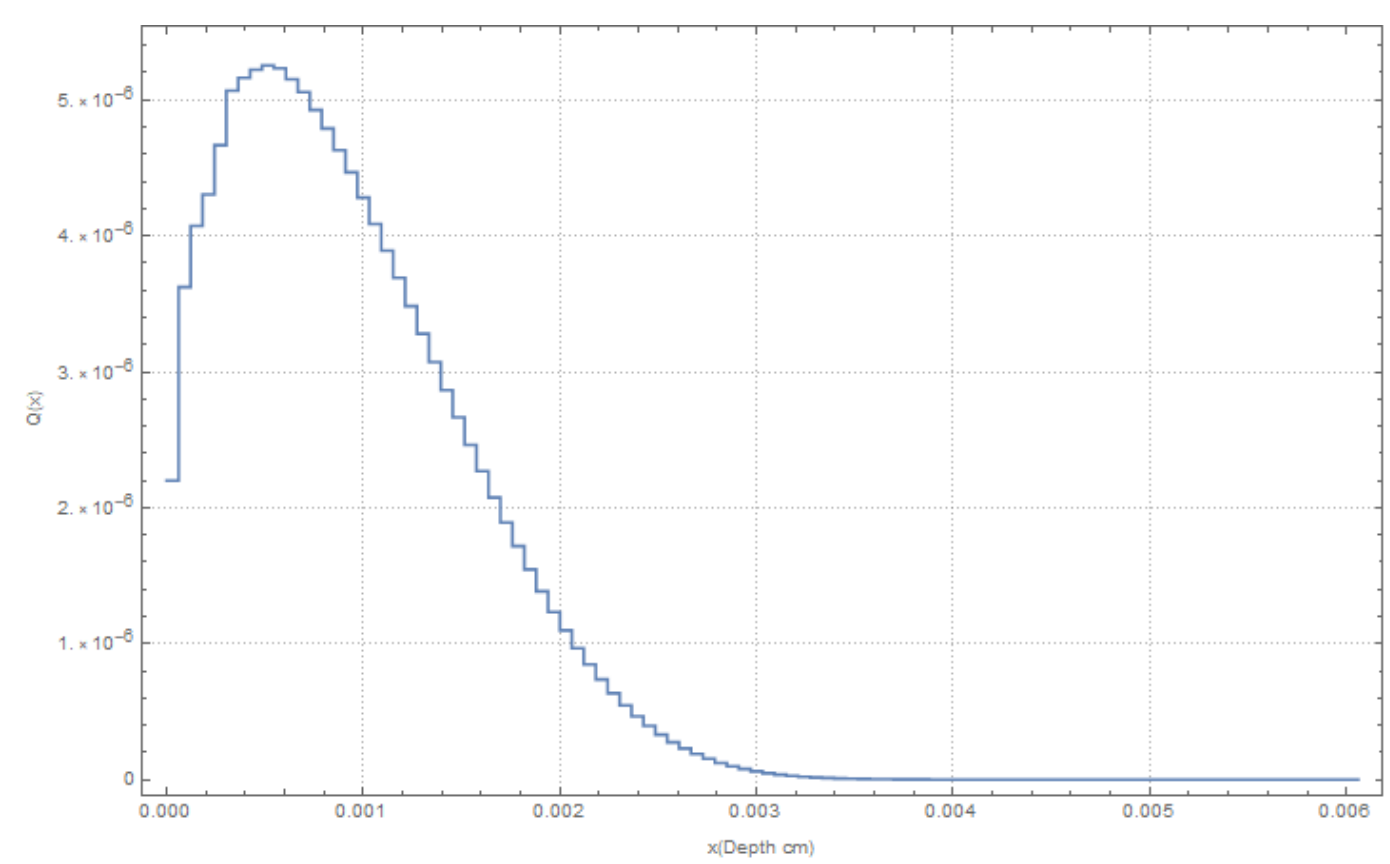

Figure 11: Final carrier distribution curve based on MCNP simulations.

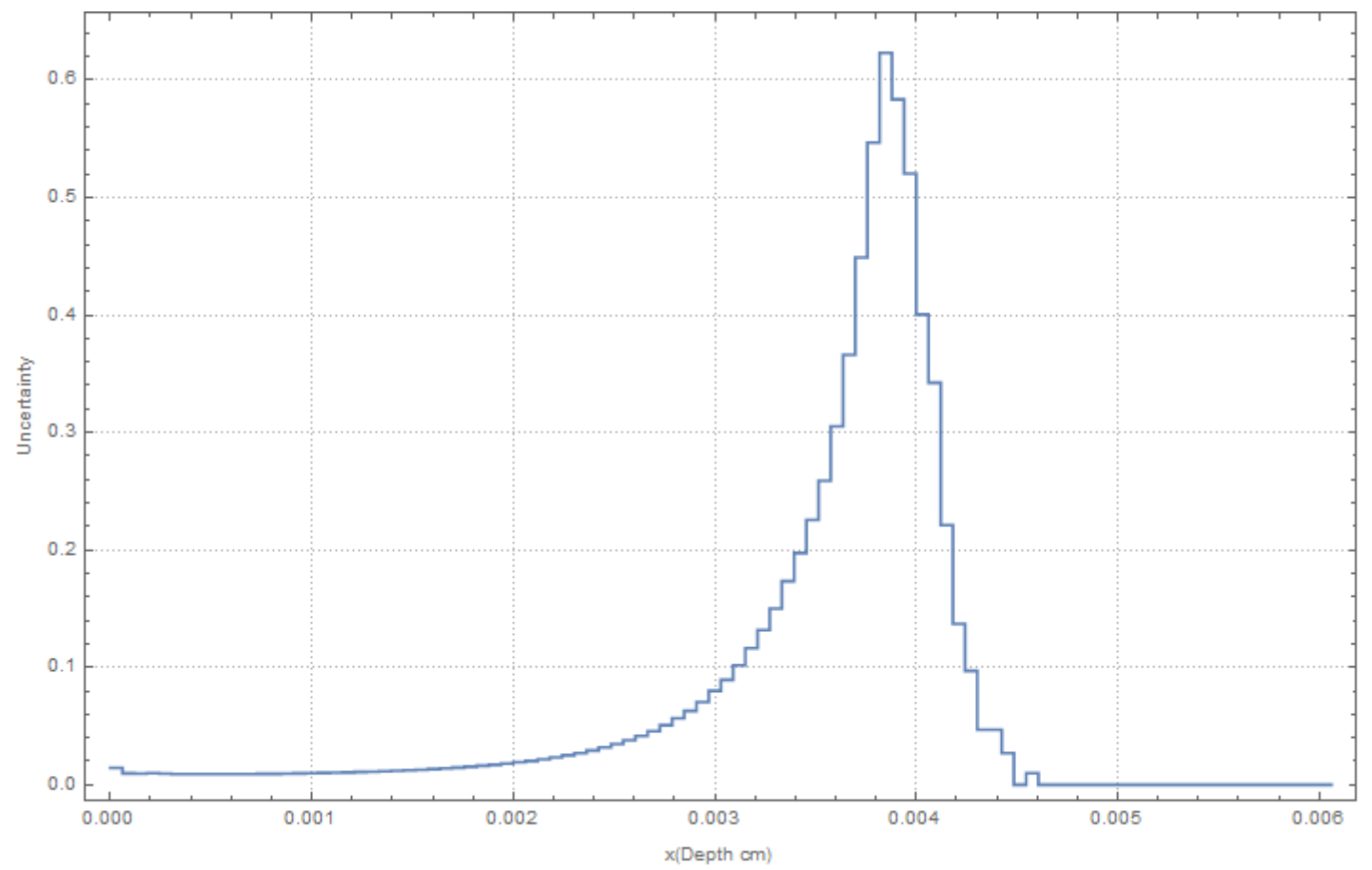

Figure 12: Associated cell uncertainties for MCNP data. During data processing cells with zero dose recorded in them had their uncertainties set to zero. This most likely explains why the curve suddenly drops off.

To transform the data into a usable form for the system of equations it is important to consider a new set of evaluations for the data. MCNP accounts for reflections and 
electrons scattering out of the medium so it was necessary to calculate what fraction of the beam energy was deposited into the volume. The energy deposition was calculated using the TMESH tally in MCNP which gives results in units of $\mathrm{MeV} /\left(\mathrm{cm}^{3}\right.$ source particle). The energy fraction deposited into the volume is then

$$
e_{\text {frac }}=\left[\sum_{i, j, k} e_{i j k}\left[\frac{\mathrm{MeV}}{\mathrm{cm}^{3} \cdot \text { source particle }}\right]\right] \times \text { Cell_Volume }\left[\mathrm{cm}^{3}\right] / \text { Electron_Energy }[\mathrm{keV}]
$$

Where $e_{i j k}$ is the energy tally in the $i j k$-th cell. Each cell has the same volume which allows the second term to be taken out of the sum. The result is still in units of energy and is then divided by the electron energy to find the total fraction of the beam energy which is deposited into the volume. If this were not a monoenergetic beam the last operation could not be used. The total energy fraction delivered into any single cell is then

$$
f_{i j k}=e_{i j k} \times\left[\sum_{i, j, k} e_{i j k}\left[\frac{\mathrm{MeV}}{\mathrm{cm}^{3} \cdot \text { source particle }}\right]\right]^{-1} \times e_{f r a c}
$$

This allows the energy deposition in any single cell to be related to the total electron beam energy: Total Energyx $f_{i j k}$. The carrier generation rate in a single cell is then easily calculated. A 1 Amp electron beam of $100 \mathrm{keV}$ electrons has a power output of $10^{5} \mathrm{~W}$. Using the $W$ value calculated previously the total carrier generation rate in any cell is

$$
\begin{aligned}
g_{i j k} & =f_{i j k} / v_{\text {cell }}\left[\mathrm{cm}^{3}\right] \times\left(10^{5} \mathrm{~W} / \mathrm{A}\right) / \mathrm{W}[4.4 \mathrm{eV} / \text { pair }] \times e_{\text {frac }} \\
& =f_{i j k} \times\left[9.64597 \times 10^{30} \frac{\text { pairs }}{\mathrm{cm}^{3} \cdot \mathrm{s} \cdot \mathrm{A}}\right]=f_{i j k} g_{0}
\end{aligned}
$$

The model can now be easily manipulated for any electron beam current. With the data in proper form the data was fitted to the following function 


$$
g(x)=\frac{1}{\sigma \sqrt{2 \pi}} \exp \left[-\frac{(x-\mu)^{2}}{2 \sigma^{2}}\right] \times \operatorname{Sech}(b x)
$$

The fitting parameters are $\{\sigma, \mu, b\}=\left\{8.43937 \times 10^{-4} \mathrm{~cm}, 5.23199 \times 10^{-3} \mathrm{~cm}, 6630.2 \mathrm{~cm}^{-1}\right\}$. The following plot is of $Q(x)$ compared to the MCNP data.

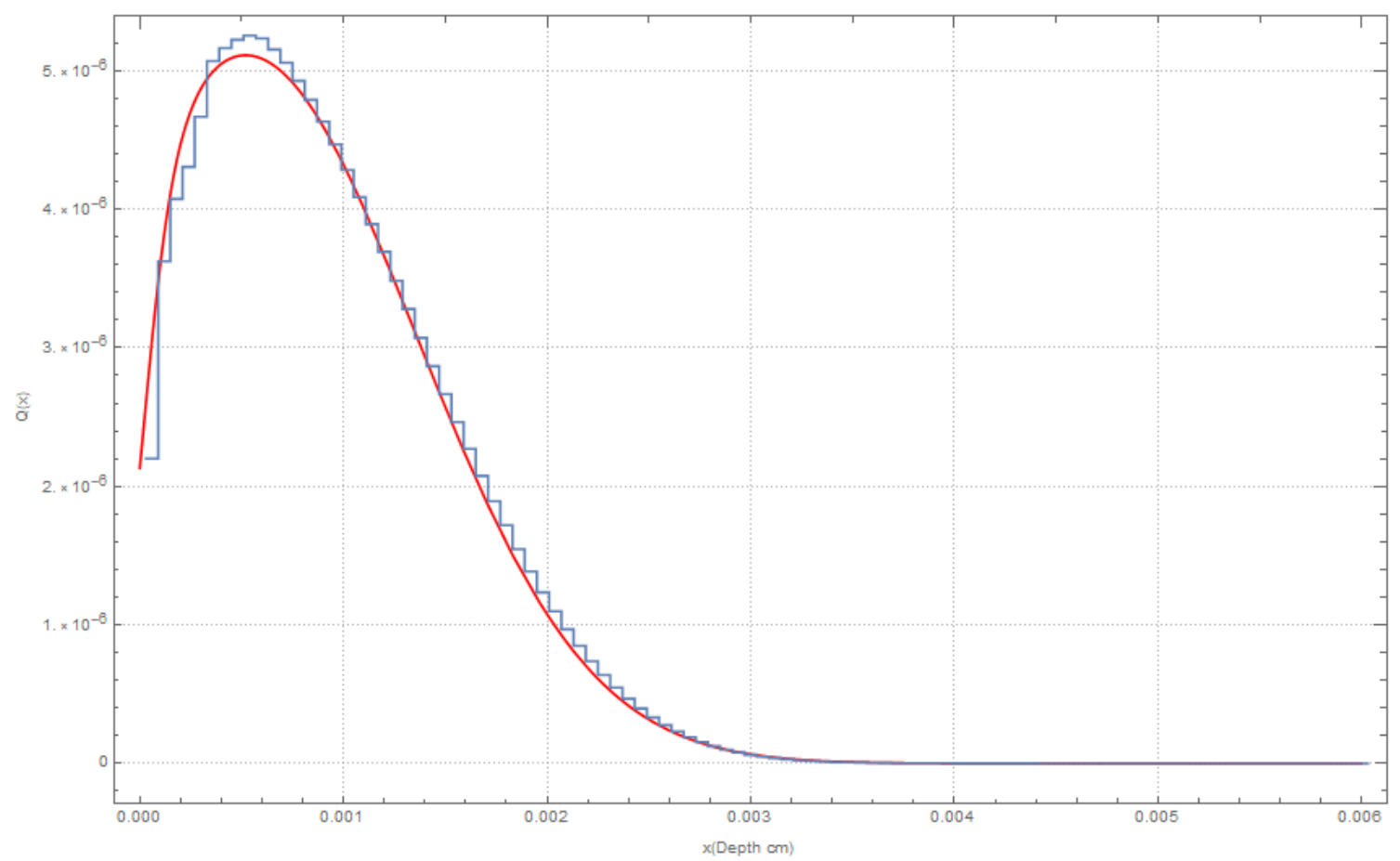

Figure 13: Comparison of MCNP data to fitted curve

\subsection{EBL Model and Results}

Before the EBL model can be solved the boundary conditions must be stated. The $\mathrm{BC}$ are as follows

$$
\begin{aligned}
& \mathcal{\varepsilon}^{\prime \prime}(\xi, 0)=0, \\
& U(0, t)=U(L k, t)=0, \\
& U(\xi, 0) \approx 0
\end{aligned}
$$


Where $L$ is some distance deep into the bulk where the electric field is assumed to be zero which is chosen to be $60 \mu \mathrm{m}$ for this study. Since equation (26) is homogeneous the boundary conditions cannot be identically zero everywhere. Equation (26) lacks a seed term found in most laser intensity equations so instead a complex seed field is set as the initial condition

$$
U(\xi, 0)=\exp (i \xi), 0<x<L k
$$

This satisfies the required IC because the average integral is zero and the expected solutions are much greater than 1 . This creates discontinuities at the boundary but when the spatial component is discretized the end result will appear to be closer to random noise.

Solving equations (26) and (27) with Mathematica's NDSolve proved to be impossible with the computational power available. So instead the problem needed to be tackled directly using finite differencing schemes. It was discovered the most effective and economical method was a semi-implicit scheme employed by Kubicek and Hlavacek for solving nonlinear boundary value problems by the method of false transient [103]. More specifically the electric field is handled semi-implicitly, while the permittivity is handled fully implicitly. It turns out that when backwards Euler differentiation is applied to equation (27) it can be solved exactly. This is especially fortuitous because this equation contains the $|U|^{2}$ term.

$$
\frac{\varepsilon_{l}^{n+1}-\varepsilon_{l}^{n}}{\Delta s}=P Q_{l}-\left(1+\delta^{2}\left|U_{l}^{n+1}\right|^{2}\right) \varepsilon_{l}^{n+1}
$$




$$
\varepsilon_{l}^{n+1}=\frac{\varepsilon_{l}^{n}+\Delta s P Q_{l}}{1+\Delta s\left(1+\delta^{2}\left|U_{l}^{n+1}\right|^{2}\right)}
$$

Where the superscripts represent the time step and the subscripts represent the spatial discretization points which run from $l=0,1,2, \ldots, m$. In equation (26) basic finite difference is employed on the derivative and only the derivative is handled implicitly, the nonlinear term is handled explicitly. That is the nonlinear terms are evaluated at the "old" time step.

$$
\begin{gathered}
\frac{2}{\omega \tau_{1}} \frac{U_{l}^{n+1}-U_{l}^{n}}{\Delta s}=i \frac{U_{l+1}^{n+1}-2 U_{l}^{n+1}+U_{l-1}^{n+1}}{\Delta \xi^{2}}+\frac{\left(1-i \chi_{1}\right) \varepsilon_{l}^{n}-\varepsilon_{0}^{\prime \prime}}{\varepsilon_{r}} U_{l}^{n} \\
\left(\frac{2}{\omega \tau_{1} \Delta s}+\frac{2 i}{\Delta \xi^{2}}\right) U_{l}^{n+1}-\frac{i}{\Delta \xi^{2}}\left(U_{l+1}^{n+1}+U_{l-1}^{n+1}\right)=\left(\frac{2}{\omega \tau_{1} \Delta s}+\frac{\left(1-i \chi_{1}\right) \varepsilon_{l}^{n}-\varepsilon_{0}^{\prime \prime}}{\varepsilon_{r}}\right) U_{l}^{n}
\end{gathered}
$$

Which alternatively could be written

$$
\left(\frac{2}{\omega \tau_{1} \Delta s}[I]-\frac{i}{\Delta \xi^{2}}[D]\right) \vec{U}^{n+1}=\vec{f}
$$

Where $[I]$ is the identity matrix, $\vec{f}$ a vector who's components make up the right hand side of equation (40) and $[D]$ is defined as

$$
[D]=\left[\begin{array}{ccccc}
-2 & 1 & & & \\
1 & -2 & 1 & & \\
& \ddots & \ddots & \ddots & \\
& & 1 & -2 & 1 \\
& & & 1 & -2
\end{array}\right]
$$

The main advantage of equation (41) is that it can be solved directly, there is no need for a newton-raphson sub-method at each time step. Additionally, there is only one 
matrix to invert which greatly speeds up computational time. In this scheme, equation (40) is evaluated first, and then equation (38) is evaluated using the resultant solutions. It is restated here the solution of interest is the field intensity

$$
I(x, t)=\varepsilon_{0} \frac{c}{2}\left|u_{0} U(x, t)\right|^{2}
$$

The first quantity sought was the threshold pump magnitude. This was found by varying the pump magnitude and observing when the first non-zero solutions appear. Figure 14 is a plot of the average integral (44) of the steady state solution vs electron beam current density

$$
I_{\text {avg }}=\frac{1}{L} \int_{0}^{L} I(x) d x
$$

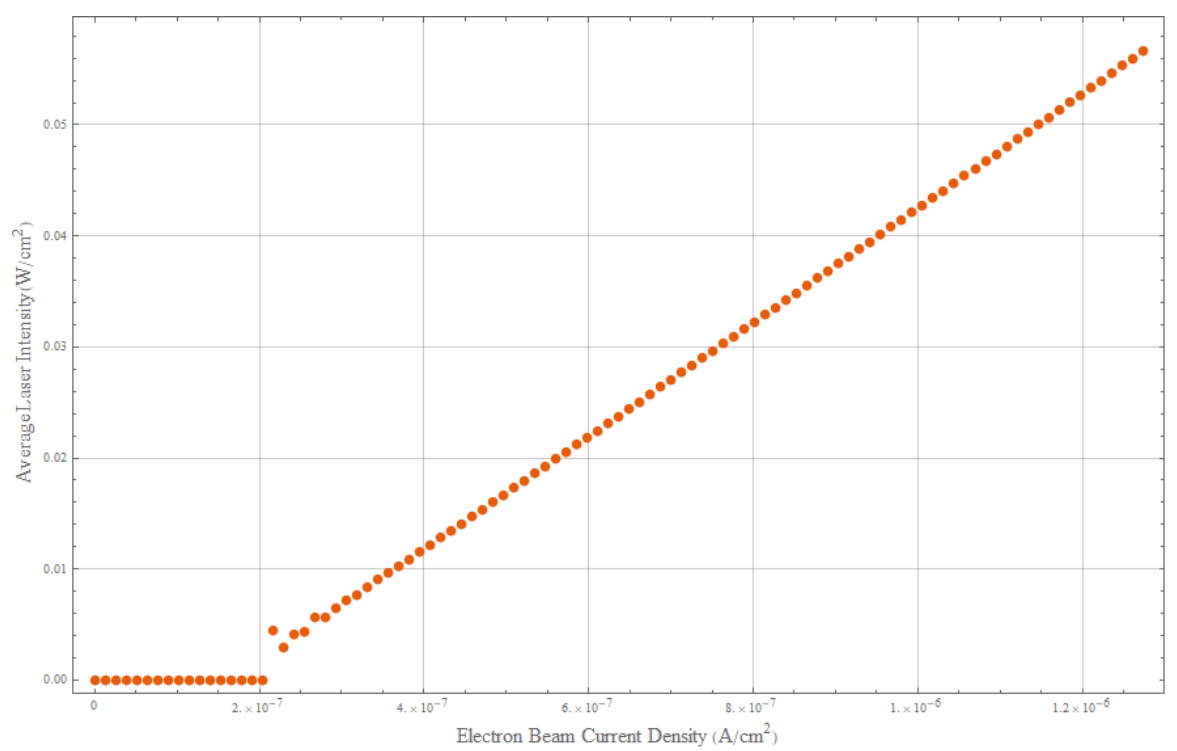

Figure 14: Plot of average laser intensity vs. electron beam current density near threshold.

From Figure 14 it is evident that, according to this model, the threshold is at a current density just above $2 \mathrm{nA} / \mathrm{cm}^{2}$. A typical plot of the field intensity over space and time is 
shown in Figure 15, a plot along the path of peak intensity and a plot of the steady state solution is given in Figure 16

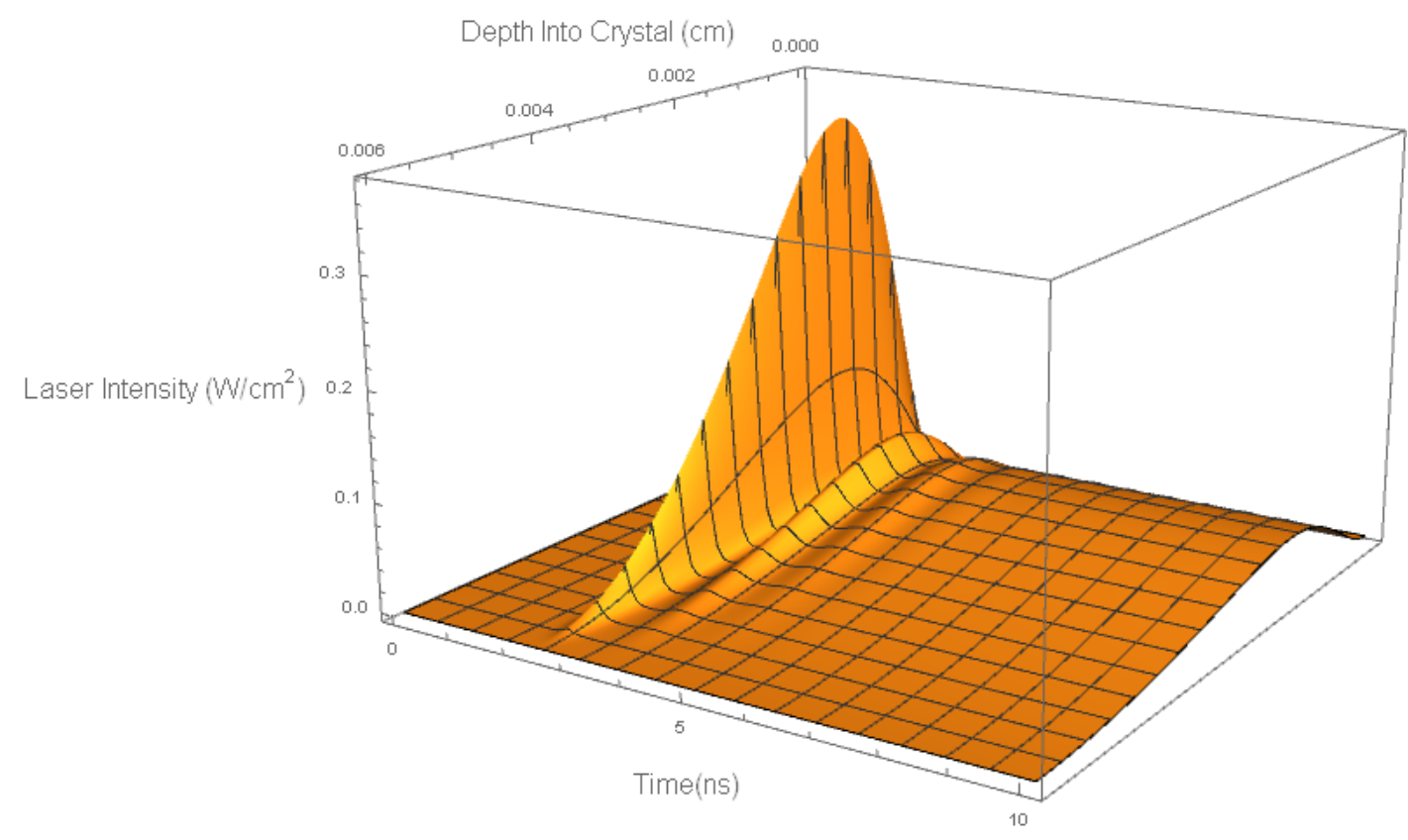

Figure 15: Full 3D Plot of the laser intensity over space and time. Data taken with $P=6.36 \mathrm{nA} / \mathrm{cm}^{2}$.
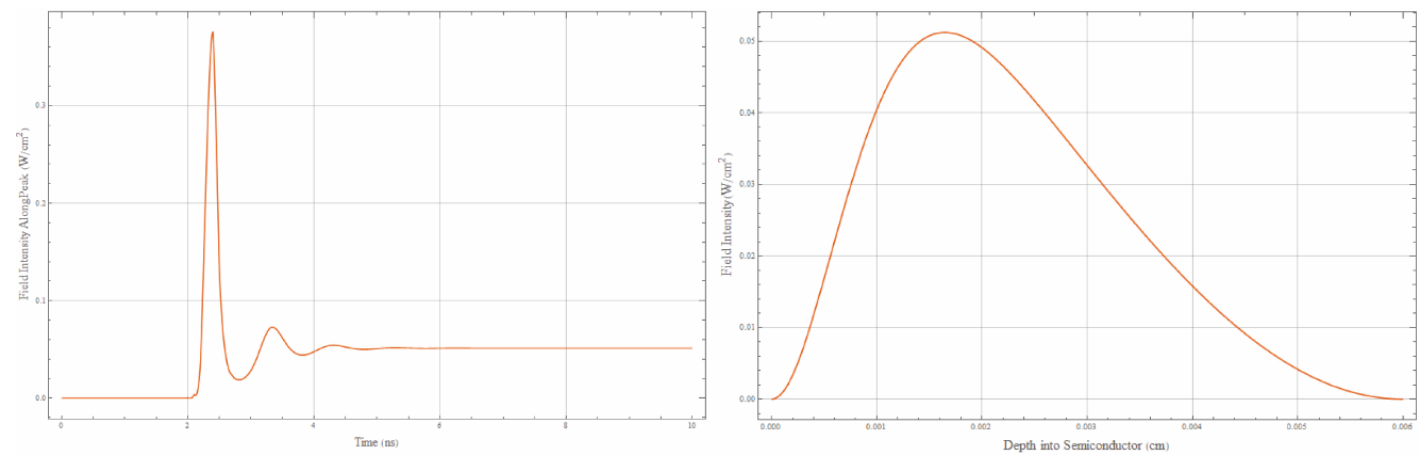

Figure 16: 1D Plot traced along the peak intensity in figure 15 (left), steady state profile (right).

It would appear from Figure 14 the average laser intensity increases linearly with electron beam current and that is indeed the case. To verify this the system of equations 
was solved for electron beam current densities well beyond threshold. Figure 17 plots the average laser intensity vs. electron beam current in the range from 1-10000 A/cm ${ }^{2}$.

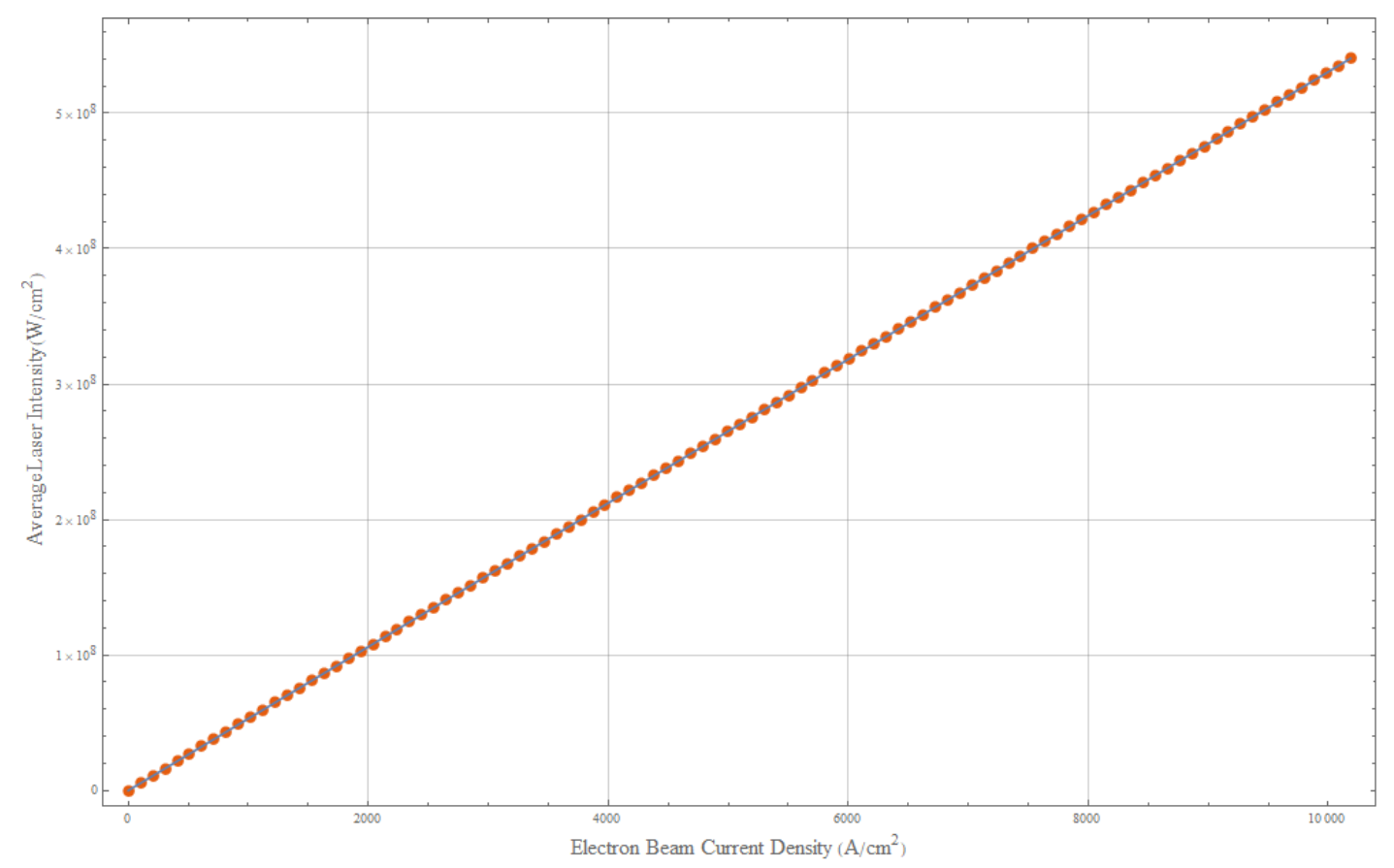

Figure 17: Average laser intensity vs. electron beam current density. The fitted line is given by equation 45

Figure 17 was fitted to an excellent degree by the linear function

$$
I_{\text {avg }}(\tilde{P}) \approx 53059.31735 \tilde{P}+0.06905086058\left[\frac{\mathrm{W}}{\mathrm{cm}^{2}}\right]
$$

A plot of a few of the solution curves is given in Figure 18 


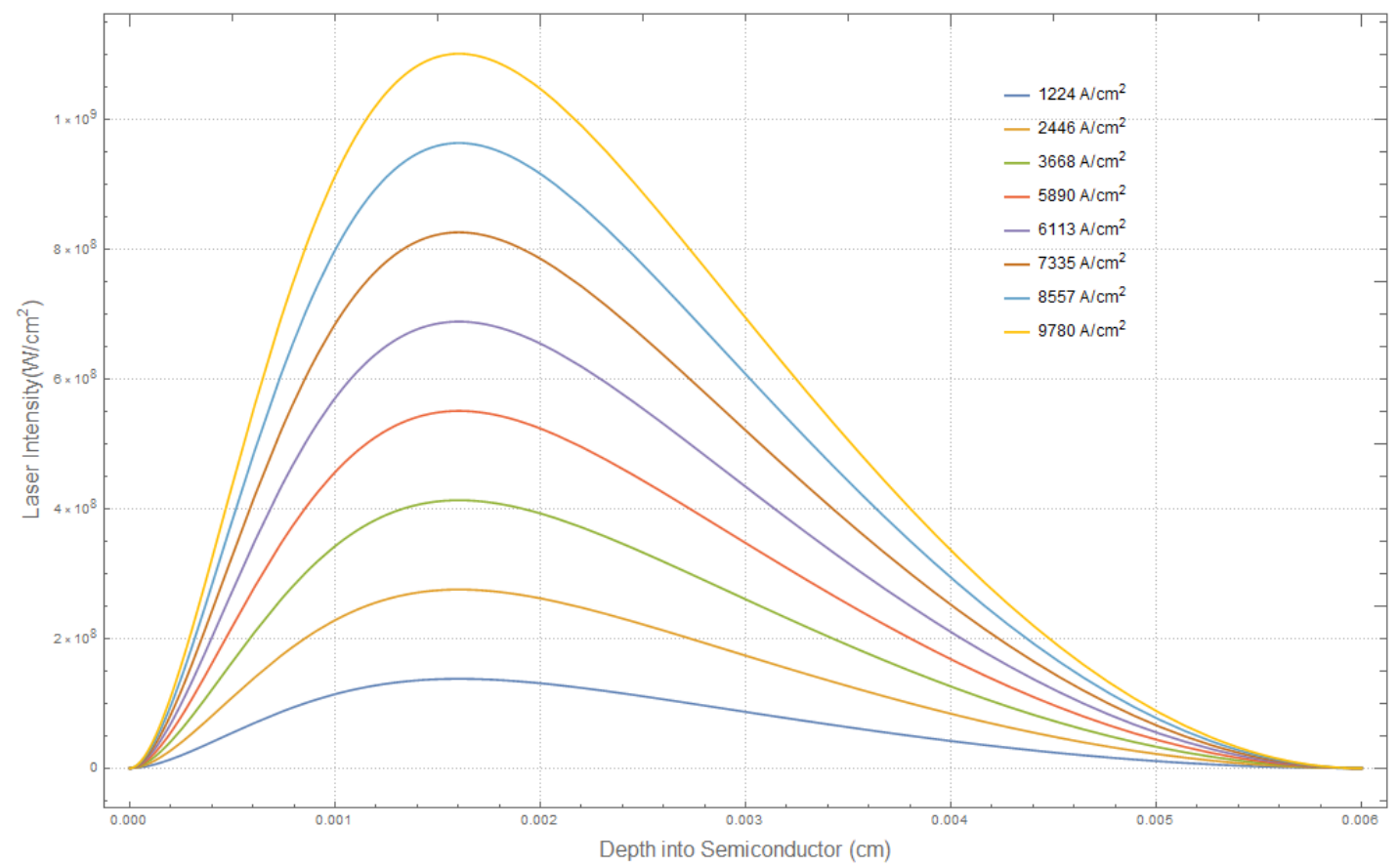

Figure 18: Steady state solution curves for laser intensity at a given electron beam intensity.

Total laser efficiency is calculated from the following

$$
\begin{aligned}
\eta(\tilde{P}) & =\frac{L \times(\text { width }) \times I_{\text {avg }}}{2 R \times(\text { width }) \times \tilde{P}}=\frac{L}{2 R} \frac{53059.31735 \tilde{P}+0.06905086058}{\left(10^{5} \mathrm{~W} / \mathrm{A}\right) \tilde{P}} \\
& \approx \frac{L}{2 R} \frac{53059.31735}{10^{5}} \approx 0.00318
\end{aligned}
$$

Where $R$ is the radius of the electron beam. This model is appropriate when the beam radius is much larger than the width of the active region under consideration.

\subsection{SSNPL Model and Results}

For the SSNPL, the same solution model is used again however the boundary conditions are different. Because the width of the semiconductor is much smaller and it is 
being pumped from both sides, Dirichlet boundary conditions are not satisfactory. It is believed the electric field cannot be considered zero at the boundaries for this problem and has been approached with Neumann boundary conditions instead.

$$
\frac{\partial U}{\partial \xi}\left(-\frac{L k}{2}, s\right)=\frac{\partial U}{\partial \xi}\left(\frac{L k}{2}, s\right)=0
$$

These conditions are handled by using $2^{\text {nd }}$ order forwards/backwards differencing

$$
\frac{\partial U}{\partial \xi}(0)=\frac{-3 U_{0}+4 U_{1}-U_{2}}{2 \Delta \xi}, \frac{\partial U}{\partial \xi}(L k)=\frac{3 U_{m}-4 U_{m-1}+U_{m-2}}{2 \Delta \xi}
$$

Which when applied to the Neumann boundary conditions yields for the end points

$$
U_{0}=\frac{1}{3}\left(4 U_{1}-U_{2}\right), U_{m}=\frac{1}{3}\left(4 U_{m-1}-U_{m-2}\right)
$$

This will modify equation (42)

$$
[D]=\left[\begin{array}{ccccc}
-\frac{2}{3} & \frac{2}{3} & & & \\
1 & -2 & 1 & & \\
& \ddots & \ddots & \ddots & \\
& & 1 & -2 & 1 \\
& & & \frac{2}{3} & -\frac{2}{3}
\end{array}\right]
$$

A table of calculated coefficients and parameters for the GaN laser is given below

Table 6: List of constants and coefficients for SSNPL model. Any constants not listed here are considered to be identical to those from the EBL table.

\begin{tabular}{|l|l|l|l|}
\hline Name & Symbol & Value & Units \\
\hline Free Carrier Lifetime & $\tau_{1}$ & $\sim 10^{-10}$ & $\mathrm{~s}$ \\
\hline
\end{tabular}




\begin{tabular}{|l|l|l|l|}
\hline Photon Frequency (3.4 eV) & $v_{0}$ & $8.22 \times 10^{14}$ & $\mathrm{~s}^{-1}$ \\
\hline$W$ Value (29) & $W$ & 10.02 & $\mathrm{eV}$ \\
\hline GaN Index of Refraction & $n$ & 2.716 & unitless \\
\hline Relative Permittivity Constant & $\varepsilon_{r}$ & 8.9 & unitless \\
\hline Natural Broadening & $\Delta v_{N}$ & $1.59 \times 10^{9}$ & $\mathrm{~s}^{-1}$ \\
\hline SE Cross Section, Lorentzian (18) & $\sigma_{L}$ & $1.66 \times 10^{-10}$ & $\mathrm{~cm}^{2}$ \\
\hline Temperature Broadening T=200 C & $\Delta v_{G}$ & $1.979 \times 10^{9}$ & $\mathrm{~s}^{-1}$ \\
\hline SE Cross Section, Gaussian (T=200 $\left.\mathrm{C}^{\circ}\right)$ & $\sigma_{G}$ & $9.55 \times 10^{-11}$ & $\mathrm{~cm}^{2}$ \\
\hline Temperature Broadening T=400 C & $\Delta v_{G}$ & $2.361 \times 10^{9}$ & $\mathrm{~s}^{-1}$ \\
\hline SE Cross Section, Gaussian $\left(\mathrm{T}=400 \mathrm{C}^{\circ}\right)$ & $\sigma_{G}$ & $9.01 \times 10^{-11}$ & $\mathrm{~cm}^{2}$ \\
\hline Electron Effective Mass & $m^{*}$ & $0.2 m_{e}$ & $\mathrm{MeV} / \mathrm{c}^{2}$ \\
\hline Intensity Coefficient (25) & $\chi_{0}$ & $2.43621 \times 10^{11}$ & $\mathrm{~s}^{-1} \mathrm{~V}^{-2}$ \\
\hline Second Relative Permittivity Coefficient $(12)$ & $\chi_{1}$ & $2.362 \times 10^{-5}$ & $\mathrm{unitless}$ \\
\hline Delta coefficient (28) & $\delta$ & $9.000 \times 10^{-5}$ & $\mathrm{unitless}$ \\
\hline
\end{tabular}

As stated previously it was assumed for simplicity the laser cavity extends the entire length of the reactor cube whose dimensions were given in Table 3.

Next the source terms must be properly defined. The distributions from Figure 6 were fitted against the following function

$$
f(x)=a \operatorname{Cosh}(b x)
$$


This was done by fitting the function against the MCNP data twice. This is allowable because the slope coefficient, $b$, depends only on the ratio of the minimum and maximum values, not on the actual minimum value. To calculate $b$ directly from the TMESH MCNP data the following equation was used

$$
\int_{-L / 2}^{L / 2} a \operatorname{Cosh}(b x) d x=\frac{2 a}{b} \operatorname{Sinh}\left(b \frac{L}{2}\right)=\sum_{i} E_{i} l_{i}
$$

Where $L$ is the depth of the semiconductor, $a$ is the average of the two minimum MCNP tally values, $E_{i}$ is the tally data from the $i_{\text {th }}$ mesh cell, and $l_{i}$ is the width of that mesh cell.

To calculate $a$ consider the total carrier distribution density of every semiconductor volume.

$$
g(x)=\frac{f_{p} f_{f} P}{V W} f(x)
$$

Where $f_{p}$ is the fraction of energy deposited by the fission fragments into the semiconductor volume, $f_{f}$ is the fraction of usable fission energy (set to $80 \%$ ), $P$ is the total power of the entire cube, $V$ is the volume of every semiconductor, and $W$ is the $W$-value for the semiconductor. Next calculate the total number of carriers generated in a single semiconductor volume

$$
(\text { width })(\text { length }) \int_{-L / 2}^{L / 2} \frac{f_{p} f_{f} P}{V W} f(x) d x=(\text { width })(\text { length }) \frac{f_{p} f_{f} P}{V W} L
$$

Where the right hand side is known immediately because the fraction $f_{p}$ was calculated directly from the $+\mathrm{F} 6$ tallies. The value for $a$ can then be calculated by the following 


$$
a=\frac{b L}{2 \operatorname{Sinh}(b L / 2)}
$$

A table contain the parameters for each thickness is given below along with the total number of cells which can fit onto one reactor core

Table 7: Source term properties for SSNPL

\begin{tabular}{|l|l|l|l|l|}
\hline Semiconductor Thickness & $3 \mu \mathrm{m}$ & $4 \mu \mathrm{m}$ & $5 \mu \mathrm{m}$ & $6 \mu \mathrm{m}$ \\
\hline$a$ coefficient & 0.838144 & 0.765892 & 0.667167 & 0.536036 \\
\hline$b$ coefficient & 6982.25 & 6492.34 & 6482.06 & 6840.97 \\
\hline Total cell number & $5 \times 10^{8}$ & $4.41 \times 10^{8}$ & $4.21875 \times 10^{8}$ & $4.2875 \times 10^{8}$ \\
\hline
\end{tabular}

The solutions sought are similar to those for the EBL case. Figure 19 shows the threshold characteristics of the laser for the 4 thicknesses with three different cross sections. The data presented is found from the formula

$$
I_{\text {total }}=A_{\text {cell }} N I_{\text {avg }}
$$

Where $A_{\text {cell }}$ is the face area of one laser cell and $N$ is the total number of laser cells in the reactor cube. Table 8 summarizes the threshold data. 

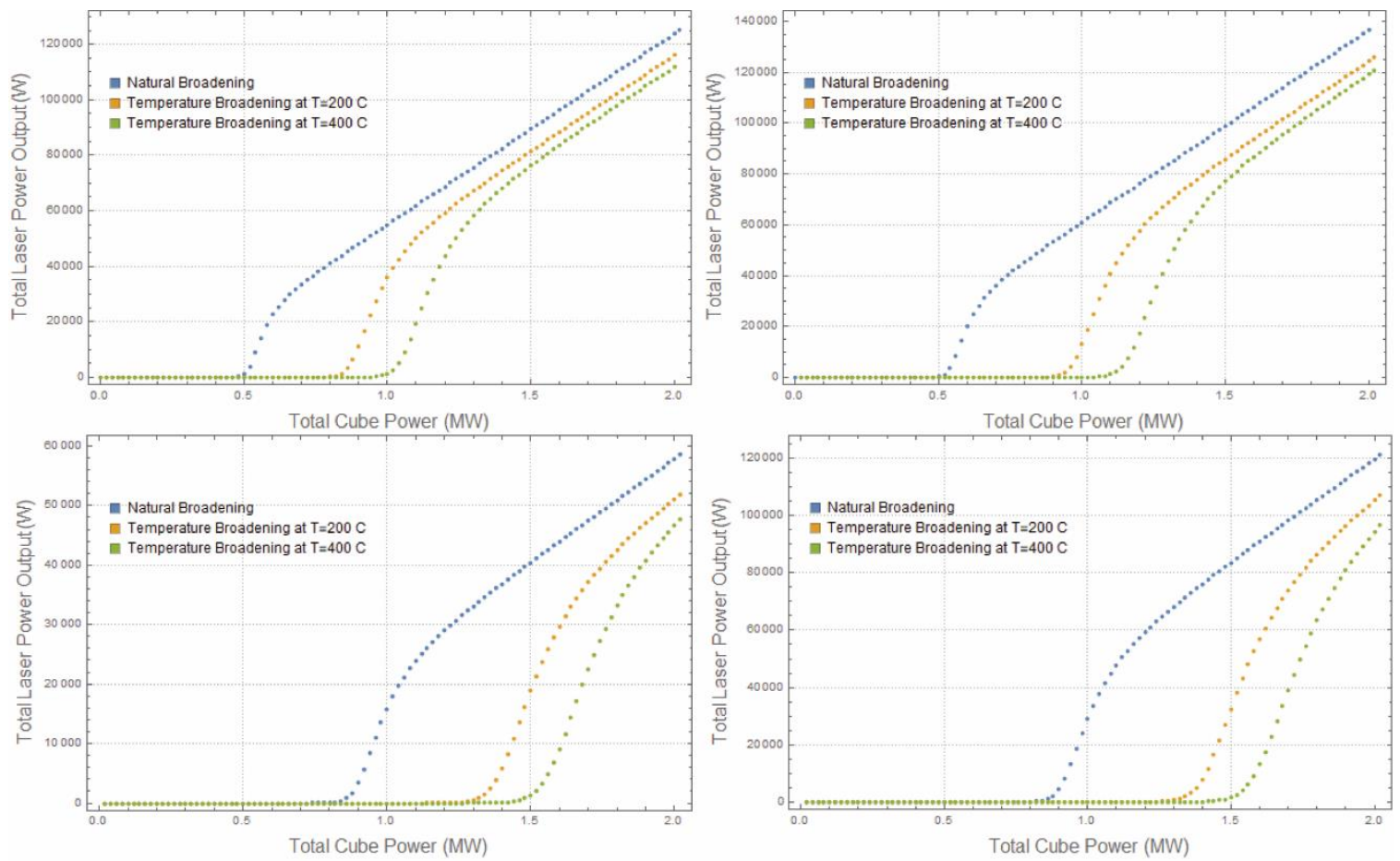

Figure 19: Threshold characteristics for SSNPL for semiconductor thicknesses $3 \mu \mathrm{m}$ (top left), $4 \mu \mathrm{m}$ (top right), 5 $\mu \mathrm{m}$ (bottom left), and $6 \mu \mathrm{m}$ (bottom right).

It is clear that as temperatures increase the threshold power increases as expected.

However, the slope of the average laser intensity slope is identical for all three forms of broadening. Meaning the maximum efficiency of the laser is unchanged with differing broadening mechanisms. The efficiency can be calculated by

$$
\eta=\frac{I_{\text {total }}}{P}
$$

The maximum efficiency can be calculated from the slope of $I_{\text {total }}$ far beyond threshold. It was calculated the SSNPL would have the highest efficiency of any NPL in existence going as high as $7.55 \%$. 
Table 8: Threshold power, power densities, and total efficiency for the laser systems

\begin{tabular}{|l|l|l|l|l|}
\hline Semiconductor Thickness & $3 \mu \mathrm{m}$ & $4 \mu \mathrm{m}$ & $5 \mu \mathrm{m}$ & $6 \mu \mathrm{m}$ \\
\hline$P_{\text {thresh }}, \sigma_{L}$ & $0.5 \mathrm{MW}$ & $0.55 \mathrm{MW}$ & $0.9 \mathrm{MW}$ & $0.9 \mathrm{MW}$ \\
\hline$P_{\text {thresh }}, \sigma_{G}(\mathrm{~T}=200 \mathrm{C})$ & $0.85 \mathrm{MW}$ & $0.95 \mathrm{MW}$ & $1.3 \mathrm{MW}$ & $1.35 \mathrm{MW}$ \\
\hline$P_{\text {thresh }}, \sigma_{G}(\mathrm{~T}=400 \mathrm{C})$ & $1 \mathrm{MW}$ & $1.1 \mathrm{MW}$ & $1.4 \mathrm{MW}$ & $1.5 \mathrm{MW}$ \\
\hline$P_{\text {density }}, \sigma_{L}$ & $7.81 \mathrm{~W} / \mathrm{cm}^{3}$ & $7.42 \mathrm{~W} / \mathrm{cm}^{3}$ & $9.88 \mathrm{~W} / \mathrm{cm}^{3}$ & $7.65 \mathrm{~W} / \mathrm{cm}^{3}$ \\
\hline$P_{\text {density }}, \sigma_{G}(\mathrm{~T}=200 \mathrm{C})$ & $13.3 \mathrm{~W} / \mathrm{cm}^{3}$ & $12.8 \mathrm{~W} / \mathrm{cm}^{3}$ & $14.3 \mathrm{~W} / \mathrm{cm}^{3}$ & $11.5 \mathrm{~W} / \mathrm{cm}^{3}$ \\
\hline$P_{\text {density }}, \sigma_{G}(\mathrm{~T}=400 \mathrm{C})$ & $15.6 \mathrm{~W} / \mathrm{cm}^{3}$ & $14.8 \mathrm{~W} / \mathrm{cm}^{3}$ & $14.4 \mathrm{~W} / \mathrm{cm}^{3}$ & $12.7 \mathrm{~W} / \mathrm{cm}^{3}$ \\
\hline$\eta_{\max }$ & 0.0687 & 0.0755 & 0.0345 & 0.0712 \\
\hline
\end{tabular}

It was found that a high gain medium was necessary to achieve threshold power levels near or below $1 \mathrm{MW}$. It is unrealistic to assume a $40 \mathrm{~cm}$ gain cavity for a semiconductor laser however this can be remedied by mirroring the output face to a reflectivity greater than $90 \%$.

The resultant solutions show a slight spatial dependence [Figure 20] however it can be shown this solution curve is very nearly flat by examining the following equation

$$
\frac{\max [I]-\min [I]}{\frac{1}{L} \int_{-L / 2}^{L / 2} I d x} \approx 6.71 \times 10^{-11}
$$



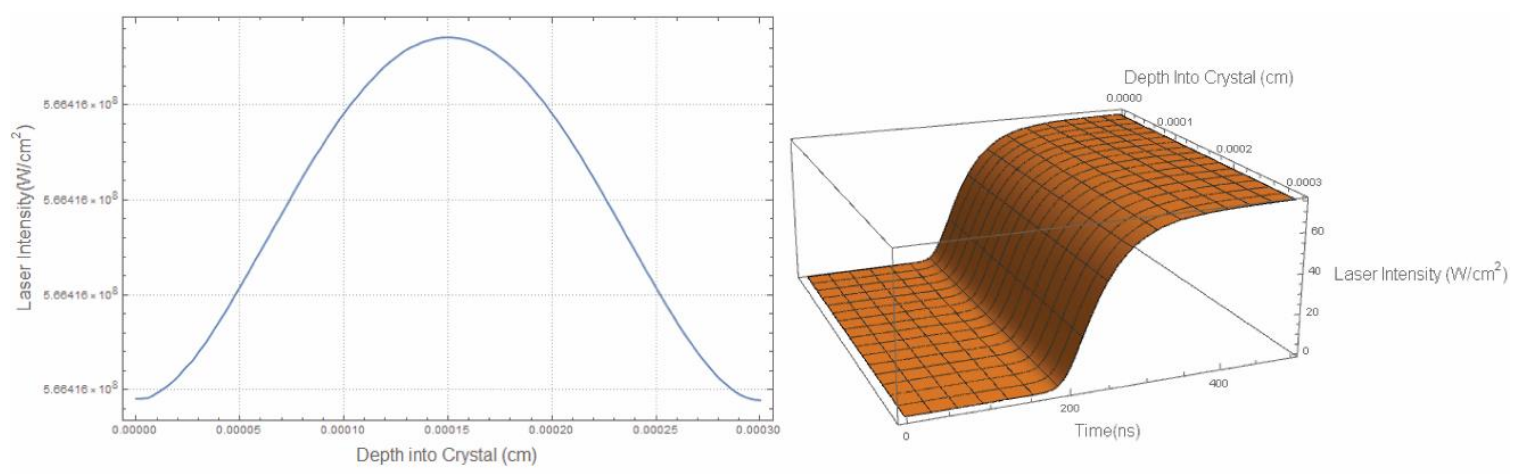

Figure 20: Plots of solution taken with $P=1 \mathrm{MW}$. Steady state profile (left), full time dependent profile (right).

It should be emphasized this analysis is based on a bare core. Adding a beryllium reflector would shrink the critical core size and threshold power considerably.

\subsection{Thermodynamic Concerns}

A nuclear pumped laser of this size would be an ideal candidate for space based laser systems. Cooling high temperature systems in space is a difficult task due to the limitations of heat transfer to the vacuum. For a long-lived orbital NPL system this would be a serious engineering difficulty, however, for this system considering the short expected lifespan of a single system sophisticated cooling systems will not be necessary. The laser cells will have suffered enough damage from the fission fragments to cease function long before temperature effects dominate. 


\section{Conclusions}

A solid state nuclear pumped laser based on alternating layers of uranium fuel and semiconductor materials has been proposed, designed, and quantified. A cost effective method for testing SSNPL systems has been proposed by analyzing the work of electron beam pumped lasers. A more generalized form of an EBL model was solved using data from MCNP. The EBL had a modest efficiency of $0.3 \%$ while the SSNPL had efficiencies in the range of 3-7\%. This would make the SSNPL the most efficient of all known NPL systems.

This work is preliminary and requires further study. Different, more robust, wide bandgap semiconductor materials, such as AlN, should be considered. Precise measurements and calculations with regard to defect formation from fission fragment impact, laser lifetime, annealing repair rate, can all serve to improve the lifetime of the system.

There are many unknowns in determining lifetime. First of all Frankel pairs can combine to form volume defects in a crystal and at room temperature about 10\% of Frankel pairs lead to agglomerate and lead to volume defects. The repair rate at elevated temperatures is unknown will be some function of temperature, $F(T)$. This means that the rate at which Frankel pairs agglomerate to form volume defects is a decreasing function of temperature, Repair Rate Function $(R R F(T))$. The second unknown is how the agglomerated Frankel pairs or volume defects impact the laser beam. The defects can scatter the beam, but since the beam is trapped by the boundaries of the solid state material, 
scattering may not be significant. The defects also could be absorption centers. The absorption cross sections for these defects are unknown and as such so are its impacts on the laser beam. This amounts to two other unknown temperature dependent functions representing scattering losses, $S L(T)$, and absorption losses, $A L(T)$. Additionally the defects formed can trap free electrons, effectively raising the lasing threshold. At elevated temperatures traps empty more quickly which can mitigate their effects to some degree.

A 90 second lifetime would make the application attractive for an antiballistic missile system or an antisatellite weapon. If the lifetime can be improved by a factor of 1000 (90000 s or $25 \mathrm{hrs),} \mathrm{then} \mathrm{the} \mathrm{system's} \mathrm{applications} \mathrm{greatly} \mathrm{expand.} \mathrm{The} \mathrm{factors} \mathrm{which}$ govern lifetime are unknown at this time. Experiments will have to run to determine $F(T)$, $\operatorname{RRF}(\mathrm{T}), \mathrm{SL}(\mathrm{T})$ and $\mathrm{AL}(\mathrm{T})$. 


\section{Appendix}

\section{A. Electron Beam Experiment}

An attempt was made to test the electron beam pumped model. An electron beam system built for basic studies was rebuilt for this experiment. The system was successfully rebuilt and was more even more consistent than its original configuration. The experiment was designed to produce a beam of $100 \mathrm{keV}$ electrons that would impact a $1 \mathrm{~cm} \times 1 \mathrm{~cm}$ square of cleaved GaAs and measure the output with a CCD camera and spectrometer. The size of the wafer was chosen because there was no guarantee how accurate the beam would be near the target and it was chosen to pick a size large enough to catch the whole beam. CST was employed to optimize the gauss coils to ensure the beam impacted the sample. It was estimated a current of 5 A was sufficient.

Unfortunately spectroscopic output was insufficient to detect to difficulties in crystal alignment with the fiberoptics and electron beam. All readings from the spectrometers in the noise level (which was considerable due to beam generation electronics and the level of electronic shielding). Some possible explanations are: Insufficient current, electron energy lower than anticipated, beam divergence before target, light pulse too short compared to the minimum integration time for the spectrometers, crystal alignment or the high noise levels from the beam electronics. 
Figure 21 is full diagram of the attempted experiment. Figure 22 is a CST simulation of the system for the electric potential and Figure 23 is the associated electron trajectories. 


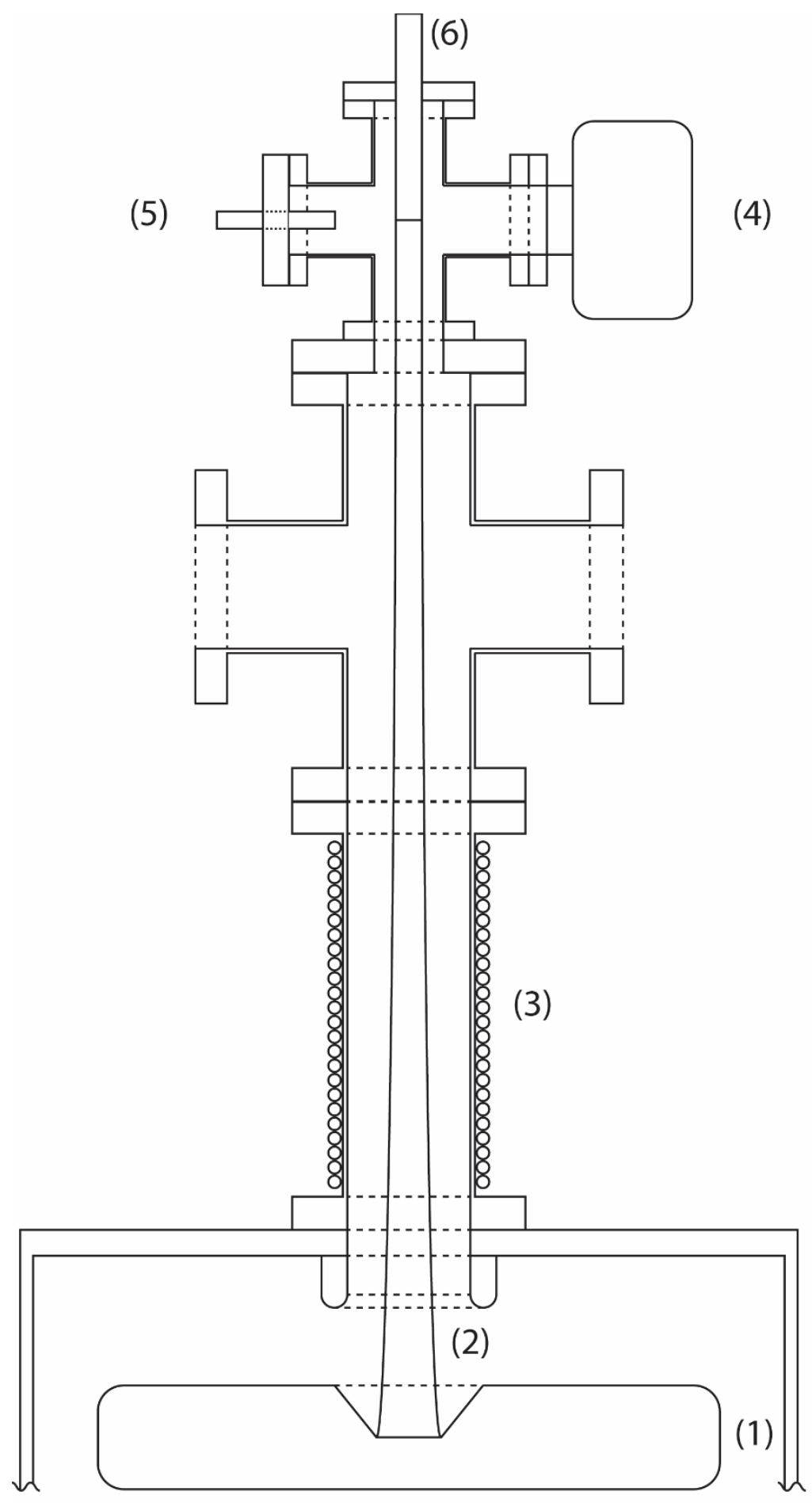

Figure 21: Full experiment setup. (1) The cathode, (2) the emitted beam, (3) the drift tube with gauss coils, (4) CCD camera, (5) Spectrometer fiber input, (6) sample holder 


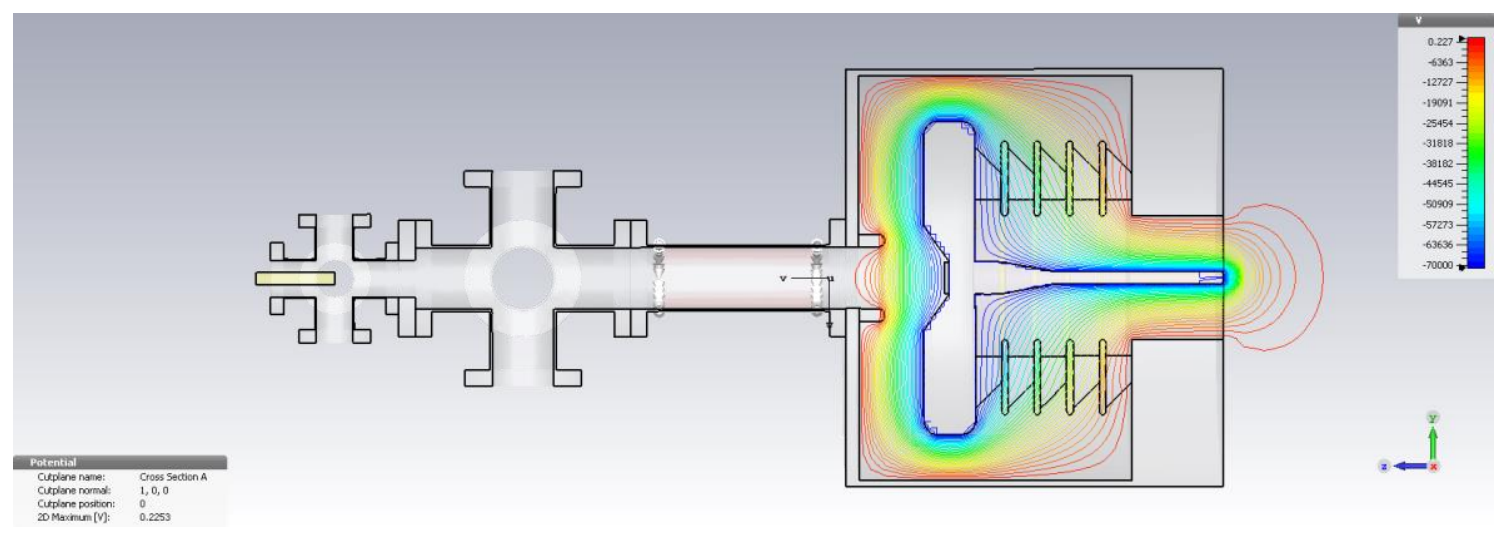

Figure 22: CST simulation showing field potential

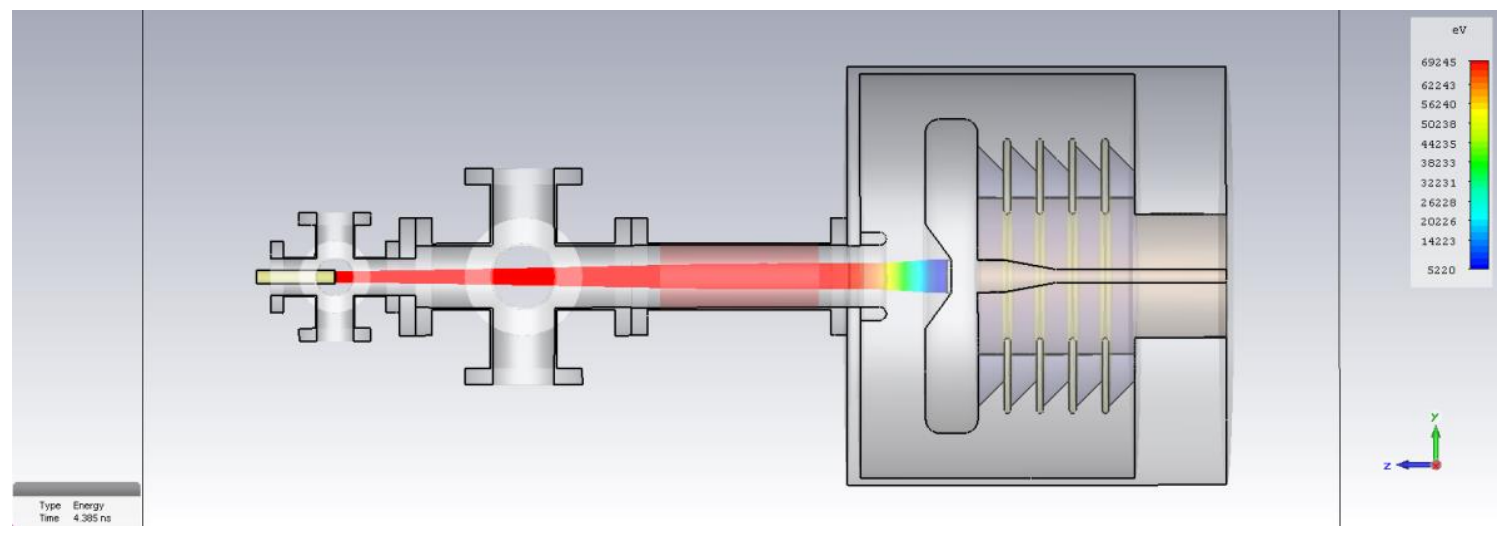

Figure 23: CST simulation showing electron trajectories

\section{B. Simplification of Permittivity Rate Equation}

It can be quickly shown that assuming $\varepsilon^{\prime \prime}(x, t) \sim g(x)$ is a zeroth order approximation.

Equation (27) is linear in $\varepsilon^{\prime \prime}$ and an exact solution can be immediately written out.

$\varepsilon^{\prime \prime}(\xi, s)=Q(\xi) \exp (-s) \exp \left(-\delta^{2} \int_{0}^{s}|U|^{2} d s^{\prime}\right) \int_{0}^{s} \exp \left(s^{\prime}\right) \exp \left(\delta^{2} \int_{0}^{s^{\prime}}|U|^{2} d s^{\prime \prime}\right) d s^{\prime}$

Next perform integration by parts repeatedly with the following method

$$
\int \frac{d f}{f x} g d x=\left.(f g)\right|_{\text {limits }}-\int f \frac{d g}{d x} d x
$$




$$
\frac{d f}{d s}=\exp (s), g=\exp \left(\delta^{2} \int_{0}^{s}|U|^{2} d s^{\prime}\right)
$$

By integrating only $\exp (s)$ and differentiating $g$ for every subsequent integration, an infinite series is constructed.

$$
\begin{aligned}
& \int_{0}^{s} \exp \left(s^{\prime}\right) \exp \left(\delta^{2} \int_{0}^{s^{\prime}}|U|^{2} d s^{\prime \prime}\right) d s^{\prime} \\
& =\exp (s)\left(\exp \left(\delta^{2} \int_{0}^{s}|U|^{2} d s^{\prime}\right)-\frac{\partial}{\partial s} \exp \left(\delta^{2} \int_{0}^{s}|U|^{2} d s^{\prime}\right)+\frac{\partial^{2}}{\partial s^{2}} \exp \left(\delta^{2} \int_{0}^{s}|U|^{2} d s^{\prime}\right)+\cdots\right) \\
& =\exp (s) \sum_{0}^{\infty}(-1)^{n} \frac{\partial^{n}}{\partial s^{n}} \exp \left(\delta^{2} \int_{0}^{s}|U|^{2} d s^{\prime}\right)
\end{aligned}
$$

Equation (59) then becomes

$$
\varepsilon^{\prime \prime}(\xi, s)=Q(\xi) \exp \left(-\delta^{2} \int_{0}^{s}|U|^{2} d s^{\prime}\right) \sum_{0}^{\infty}(-1)^{n} \frac{\partial^{n}}{\partial s^{n}} \exp \left(\delta^{2} \int_{0}^{s}|U|^{2} d s^{\prime}\right)
$$

If $n=0$ then $\varepsilon^{\prime \prime}$ is simply $Q(\xi)$. This assumption essentially states the field does not appreciably effect the carrier distribution shape, a weak pumping condition.

\section{Criticality MCNP Code}

The MCNP code used to calculate the criticality of the core is below.

Criticality calculation for homogeneous laser system

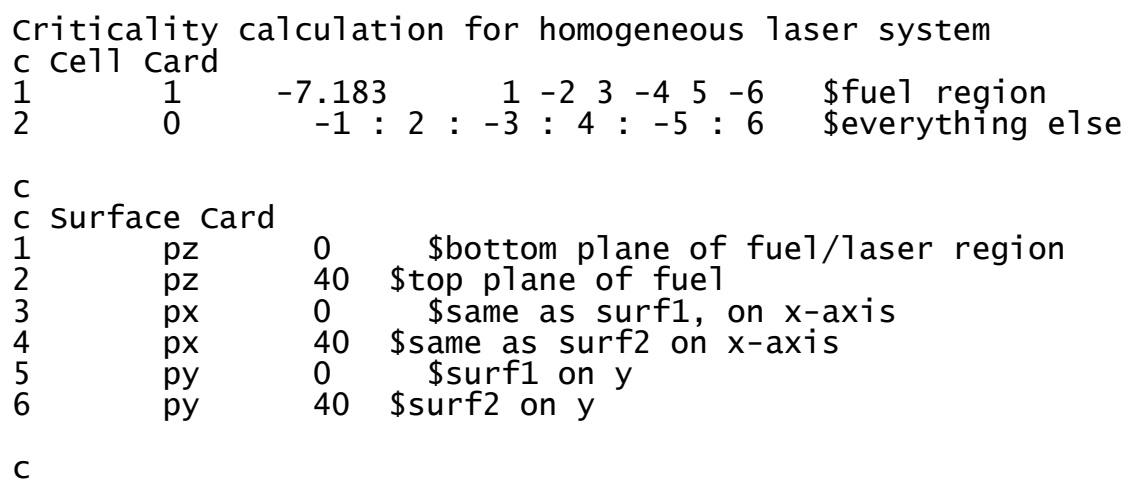




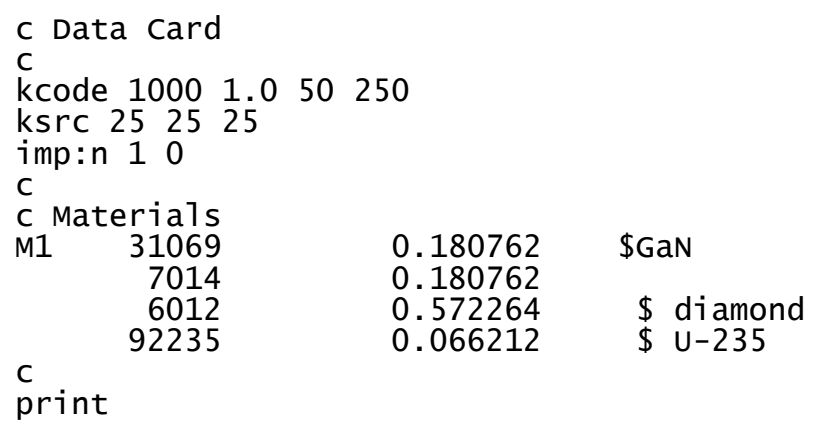

\section{Fission Product Transport Code}

Example code used to transport fission products. Note that in the mesh tally the first and last mesh points are inside the uranium. For unresolved reasons the energy distribution across the mesh would not be uniform unless it was written this way. It is the middle 16 mesh data points which are used for the SSNPL model.

MESSAGE : MCTAL=36089_1cm_3cm_MCTAL.txt NAME=36089_1cm_3_dose.

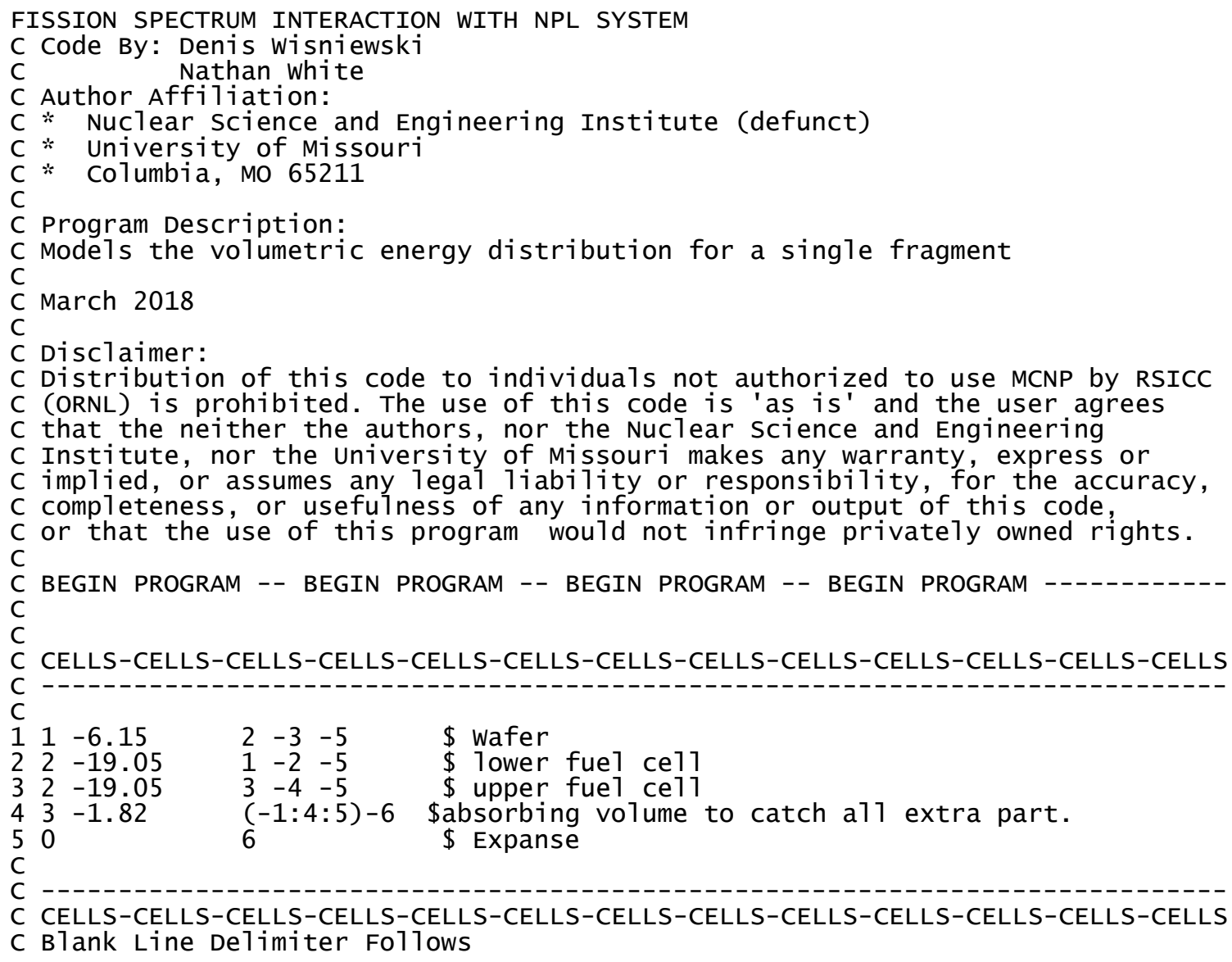




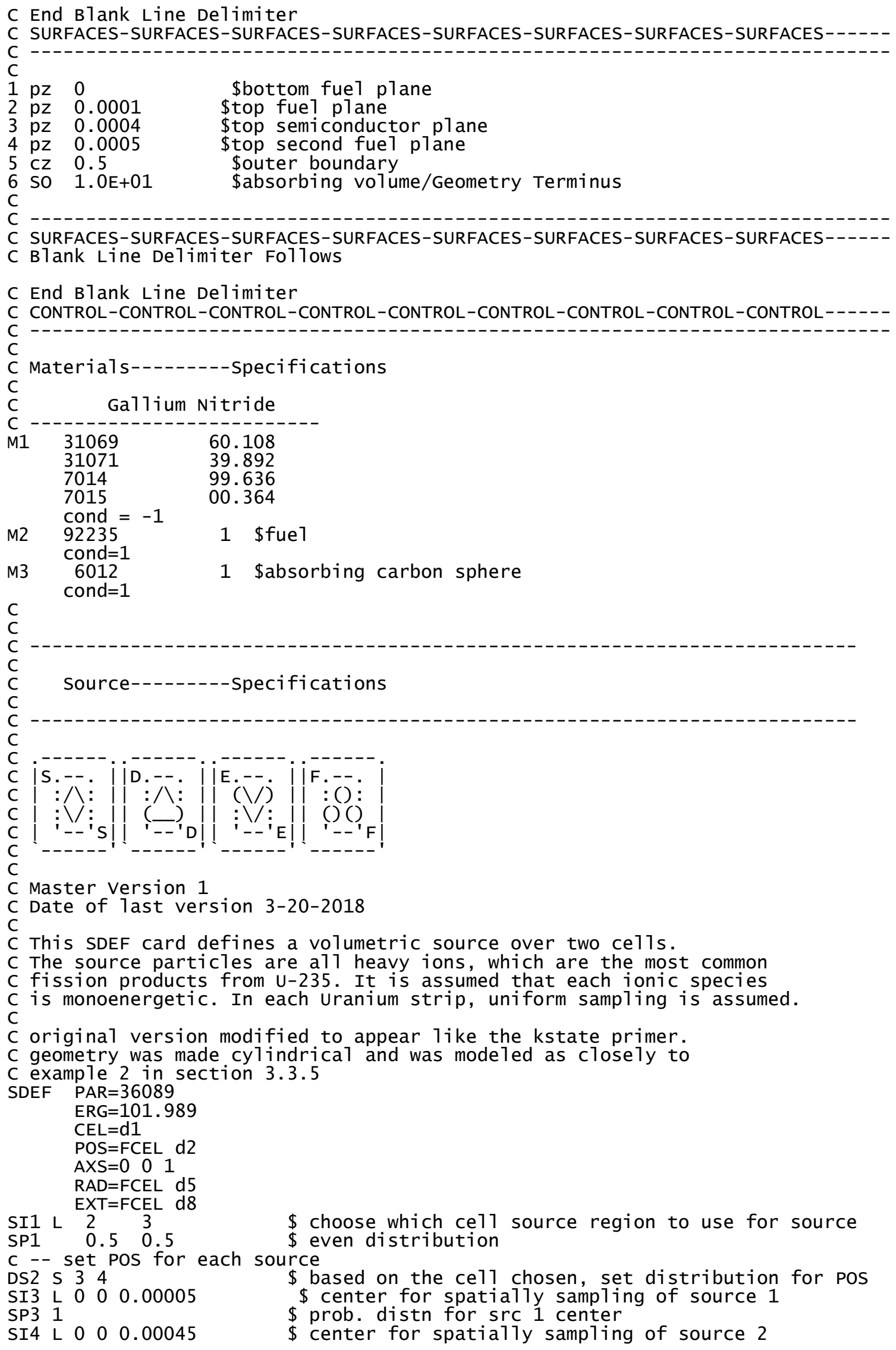




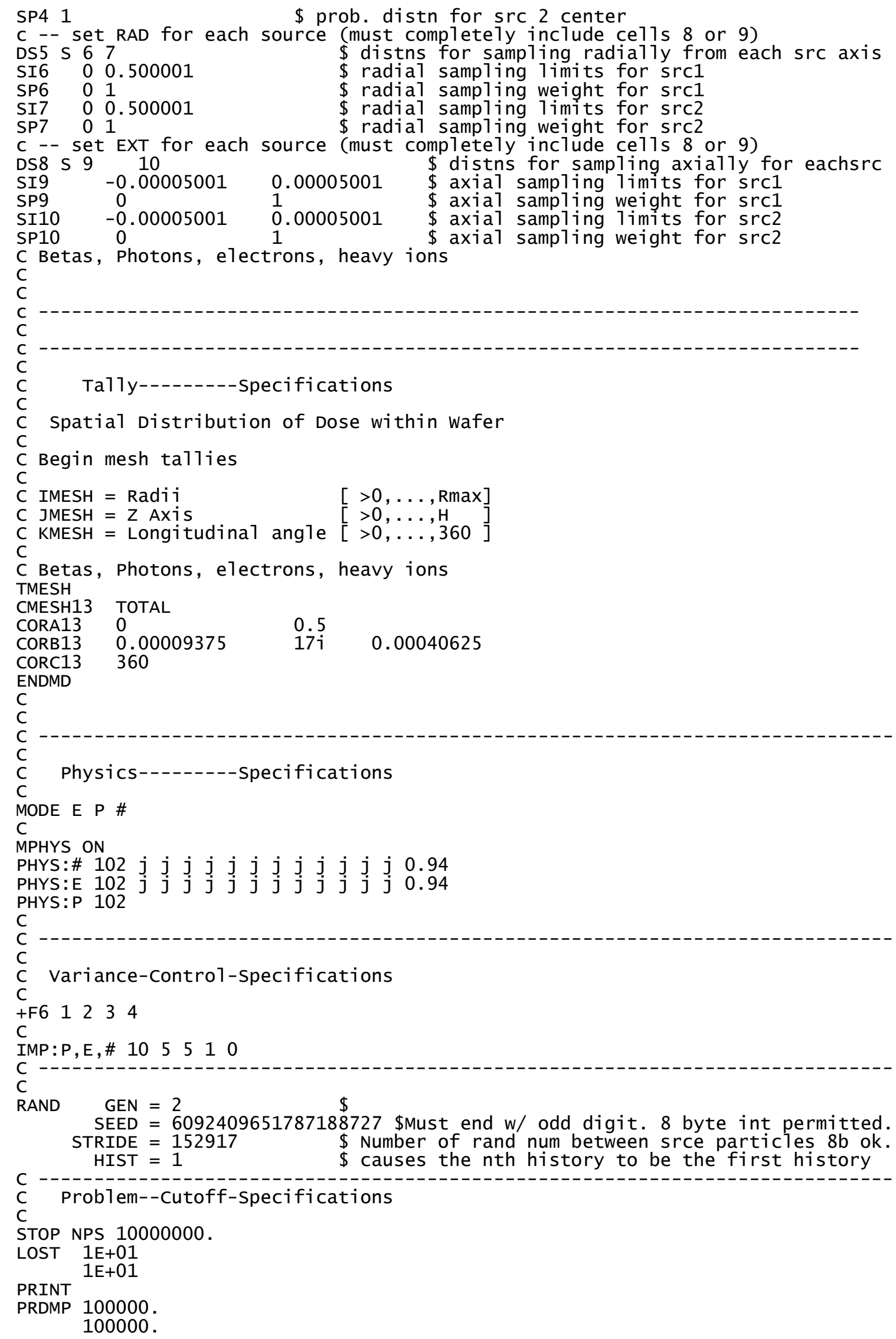




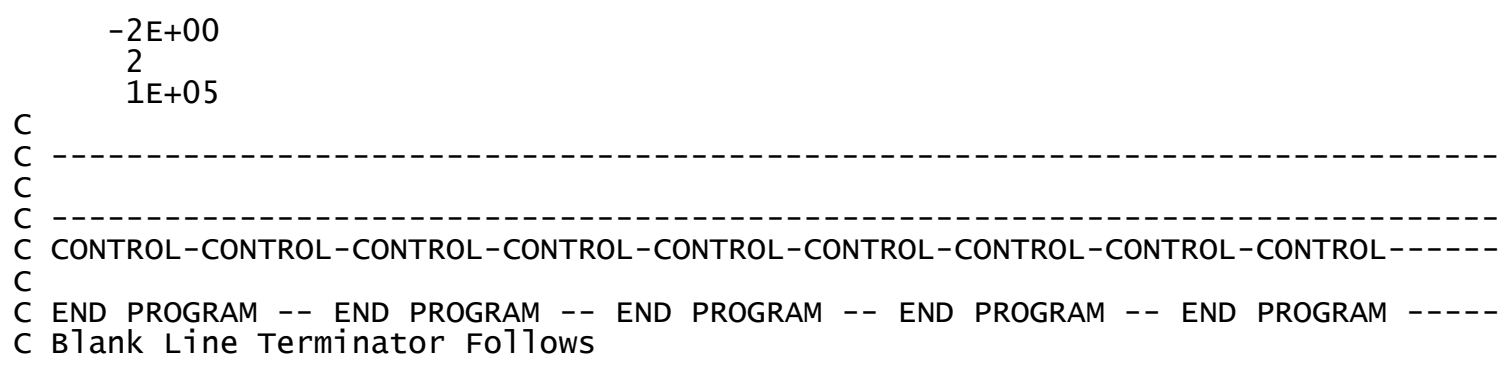




\section{References}

[1] M. Prelas, Nuclear-Pumped Lasers: Springer International Publishing, 2015.

[2] M. A. Prelas, M. L. Watermann, D. A. Wisniewski, J. A. Neher, and C. L. Weaver Iii, "A review of nuclear pumped lasers and applications (asteroid deflection)," in ASEE Annual Conference and Exposition, Conference Proceedings, 2014.

[3] C. Yang, H. Chen, C. Zheng, X. Zhao, and H. Han, "The progress of nuclear pumped laser in CFBR-II reactor," Chin. Opt. Lett., vol. 1, pp. 292-291, 2003.

[4] E. P. Magda, "Powerful nuclear-pumped lasers: a review," in Twelfth International Symposium on Gas Flow and Chemical Lasers and High-Power Laser Conference, 1998, pp. 93-103.

[5] A. A. Sinyanskii and S. P. Melnikov, "Research on development of continuous nuclear-laser setups in VNIIEF," Proceedings of SPIE - The International Society for Optical Engineering, vol. 3686, pp. 43-55, 1999.

[6] Y. A. Dyuzhov, O. F. Kukharchuk, E. D. Poletaev, V. N. Smolsky, and A. A. Suvorov, "Lasing characteristics of the Ar-Xe and He -Ar -Xe mixtures pumped by fission fragments," Quantum Electronics, vol. 40, pp. 11-18, 2010.

[7] A. Konak, S. Mel'nikov, V. Porkhaev, and A. Sinyanskii, "Characteristics of nuclear-pumped lasers based on the $3 \mathrm{p}-3 \mathrm{~s}$ transitions in the neon atom," Quantum Electronics, vol. 25, p. 209, 1995.

[8] A. A. Pikulev, S. V. Patyanin, A. A. Sinyanskii, P. V. Sosnin, S. L. Turutin, and V. M. Tsvetkov, "Forming of optical radiation of multi-channel nuclear-pumped lasers," 2007, pp. 69380D-69380D-10.

[9] A. V. Zagidulin, A. V. Bochkov, V. V. Mironenko, and G. S. Sofienko, "A 500-J nuclear-pumped gas laser," Technical Physics Letters, vol. 38, pp. 1059-1062, 2012.

[10] A. B. Carter, "Directed energy missile defense in space," NASA STI/Recon Technical Report N, vol. 85, 1984.

[11] A. Gibbings, M. Vasile, J.-M. Hopkins, D. Burns, and I. Watson, "Potential of laserinduced ablation for future space applications," Space Policy, vol. 28, pp. 149-153, 2012/08/01 2012. 
[12] C. R. Phipps and J. R. Luke, "Laser Space Propulsion," in Laser Ablation and its Applications, C. Phipps, Ed., ed Boston, MA: Springer US, 2007, pp. 407-434.

[13] T. E. Repetti, "Application of reactor-pumped lasers to power beaming," DTIC Document 1991.

[14] C. R. Phipps, G. Albrecht, H. Friedman, D. Gavel, E. V. George, J. Murray, et al., "ORION: Clearing near-Earth space debris using a 20-kW, 530-nm, Earth-based, repetitively pulsed laser," Laser and Particle Beams, vol. 14, pp. 1-44, 1996/003/001 1996.

[15] D. A. McArthur and P. B. Tollefsrud, "Observation of laser action in CO gas excited only by fission fragments," Applied Physics Letters, vol. 26, pp. 187-190, 1975.

[16] H. H. Helmick, J. L. Fuller, and R. T. Schneider, "Direct nuclear pumping of a helium-xenon laser," Applied Physics Letters, vol. 26, pp. 327-328, 1975.

[17] A. M. Voinov, L. E. Dovbyshov, V. N. Krivonosov, S. P. Mel'nikov, I. V. Podmoshenskii, and A. A. SinyanskiiI, "Nuclear-Pumped IR Lasers Using ArI, KrI, and XeI Transitions," Soviet Phys. Tech. Phys. Lett., vol. 5, 1979.

[18] W. J. Alford and G. N. Hays, "Measured laser parameters for reactor-pumped He/Ar/Xe and Ar/Xe lasers," Journal of Applied Physics, vol. 65, pp. 3760-3766, 1989.

[19] G. A. Hebner and G. N. Hays, "Fission-fragment excited xenon/rare gas mixtures. I. Laser parameters of the $1.73 \mu \mathrm{m}$ xenon transition," Journal of Applied Physics, vol. 73, pp. 3614-3626, 1993.

[20] R. J. DeYoung, W. E. Wells, G. H. Miley, and J. T. Verdeyen, "Direct nuclear pumping of a Ne-N2 laser," Applied Physics Letters, vol. 28, pp. 519-521, 1976.

[21] M. A. Akerman, G. H. Miley, and D. A. McArthur, "A helium-mercury direct nuclear pumped laser," Applied Physics Letters, vol. 30, pp. 409-412, 1977.

[22] M. A. Prelas, M. A. Akerman, F. P. Boody, and G. H. Miley, "A direct nuclear pumped 1.45- $\mu$ atomic carbon laser in mixtures of He-CO and He-CO2," Applied Physics Letters, vol. 31, pp. 428-430, 1977.

[23] N. W. Jalufka, R. J. DeYoung, F. Hohl, and M. D. Williams, "Nuclear-pumped ${ }^{3} \mathrm{He}-$ Ar laser excited by the ${ }^{3} \mathrm{He}(\mathrm{n}, \mathrm{p})^{3} \mathrm{H}$ reaction," Applied Physics Letters, vol. 29, pp. 188-190, 1976.

[24] R. J. De Young, N. W. Jalufka, and F. Hohl, "Nuclear-pumped lasing of ${ }^{3} \mathrm{He}-\mathrm{Xe}$ and ${ }^{3} \mathrm{He}-\mathrm{Kr}, "$ Applied Physics Letters, vol. 30, pp. 19-21, 1977.

[25] R. J. DeYoung, N. W. Jalufka, and F. Hohl, "Direct Nuclear-Pumped Lasers Using the ${ }^{3} \mathrm{He}(\mathrm{n}, \mathrm{p})^{3} \mathrm{H}$ Reaction," AIAA Journal, vol. 16, pp. 991-998, 1978/09/01 1978. 
[26] Y. R. Shaban and G. H. Miley, "Practical, visible wavelength nuclear-pumped laser," Laser and Particle Beams, vol. 11, pp. 559-566, 1993/009/001 1993.

[27] W. H. Williams and G. H. Miley, "Lasing in atomic iodine with a nuclear-pumped XeBr* flash lamp," Applied Physics Letters, vol. 62, pp. 1724-1726, 1993.

[28] M. A. Prelas and S. K. Loyalka, "A review of the utilization of energetic ions for the production of excited atomic and molecular states and chemical synthesis," Progress in Nuclear Energy, vol. 8, pp. 35-52, 1981/01/01 1981.

[29] H. A. Hassan, "Analysis of the UF6-Xe Direct Nuclear-Pumped Laser," AIAA Journal, vol. 18, pp. 1490-1494, 1980/12/01 1980.

[30] M. Prelas, Nuclear Pumped Lasers. New York: Springer International, 2016.

[31] F. P. Boody, M. A. Prelas, J. H. Anderson, S. J. Nagalingam, and G. H. Miley, "Progress in nuclear-pumped lasers," Progress in Astronautics and Aeronautics, vol. 61, pp. 379-410, 1978.

[32] G.-X. Gu, J. F. Kunze, F. P. Boody, and M. A. Prelas, "Neutronics conceptual design of a UF6-fueled gasesous laser system," in Transactions of the Fifth Symposium on Space Nuclear Power Systems, 1988, pp. 149-152.

[33] R. J. Rodgers, U. S. N. Aeronautics, S. A. Scientific, T. I. Office, and L. R. Center, Initial conceptual design study of self-critical nuclear pumped laser systems: National Aeronautics and Space Administration, Scientific and Technical Information Office, 1979.

[34] M. A. Prelas, F. P. Boody, and M. S. Zediker, "An aerosol core nuclear reactor for space-based high energy/power nuclear-pumped lasers," Space Nuclear Power Systems, vol. 4, 1985.

[35] D. J. Mencin and M. A. Prelas, "Gaseous like uranium reactors at low temperatures using C60 cages," Proceedings of Nuclear Technologies for Space Exploration, 1992.

[36] X. Liu, L. Li, B. Liu, D. Wang, Y. Zhao, and X. Gao, "Theoretical Study on the Ground State Structure of Uranofullerene U@C82," The Journal of Physical Chemistry A, vol. 116, pp. 11651-11655, 2012/11/29 2012.

[37] R. J. Deyoung, N. W. Jalufka, and F. Hohl, "Nuclear-pumped lasing of 3He-Xe and 3He-Kr," Applied Physics Letters, vol. 30, pp. 19-21, 1977.

[38] A. Miskevich, A. Dmitriev, V. Iliashchenko, B. Salamakha, V. Stepanov, and E. Gorodkov, "Lasing in $\mathrm{Cd}$ vapor pumped by products of the nuclear reaction $\mathrm{He}-$ 3/n, p/T," Technical Physics Letters, vol. 6, p. 352, 1980. 
[39] W. H. Williams and G. H. Miley, "Lasing in atomic iodine with a nuclear-pumped XeBr* flash lamp," Applied Physics Letters, vol. 62, pp. 1724-1726, 1993.

[40] Y. Shaban and G. Miley, "Practical, visible wavelength nuclear-pumped laser," Laser and Particle Beams, vol. 11, pp. 559-566, 1993.

[41] M. A. Prelas, "Nuclear-driven solid-state lasers," in Lasers' 89, 1990, pp. 263-269.

[42] M. A. Prelas, F. P. Boody, G. H. Miley, and J. F. Kunze, "Nuclear driven flashlamps," Laser and Particle Beams, vol. 6, pp. 25-62, 1988.

[43] F. Boody and M. Prelas, "Very High Average Power Solid-State Lasers Pumped by Remotely-Located Nuclear-Driven Fluorescers," Advanced Solid-State Lasers, pp. 192-199, 1990.

[44] P. Ebert, L. Ferderber, H. Koehler, R. Kuckuck, and D. Redhead, "Amplified spontaneous emission in xenon pumped by gamma rays," Quantum Electronics, IEEE Journal of, vol. 10, pp. 736-736, 1974.

[45] P. Lyons, J. Clarke, and D. Metzger, "Gamma initiated HF laser," Quantum Electronics, IEEE Journal of, vol. 10, pp. 736-736, 1974.

[46] J. Hecht, "The Strange Saga of the X-Ray Laser," in Beam Weapons, ed: Springer US, 1984, pp. 115-139.

[47] O. Bogdankevich, "Electron-beam-pumped semiconductor lasers," Quantum Electronics, vol. 24, p. 1031, 1994.

[48] C. E. Hurwitz, "EFFICIENT ULTRAVIOLET LASER EMISSION IN ELECTRON-BEAM-EXCITED ZnS," Applied Physics Letters, vol. 9, pp. 116$118,1966$.

[49] C. E. Hurwitz, "EFFICIENT VISIBLE LASERS OF CdS $\mathrm{CSe}_{1-\mathrm{x}}$ BY ELECTRONBEAM EXCITATION," Applied Physics Letters, vol. 8, pp. 243-245, 1966.

[50] V. Daneu, D. DeGloria, A. Sanchez, F. Tong, and R. Osgood Jr, "Electron-pumped high-efficiency semiconductor laser," Applied physics letters, vol. 49, pp. 546-548, 1986.

[51] C. E. Hurwitz, "HIGH POWER AND EFFICIENCY IN CdS ELECTRON BEAM PUMPED LASERS," Applied Physics Letters, vol. 9, pp. 420-423, 1966.

[52] V. Kozlovskiı̌, A. S. Nasibov, and P. Reznikov, "Investigation of cw operation of a GaAs laser pumped by an electron beam," Quantum Electronics, vol. 12, pp. 14371440, 1982. 
[53] O. Bogdankevich, "The use of electron-beam pumped semiconductor lasers in projection television," Quantum Electronics, IEEE Journal of, vol. 14, pp. 133-135, 1978.

[54] O. Bogdankevich, N. Borisov, A. N. Georgobiani, V. Gutan, Z. Ilyukhina, B. Lavrushin, et al., "Continuous tuning of the emission frequency of electron-beampumped lasers," Soviet Journal of Quantum Electronics, vol. 5, p. 1215, 1975.

[55] A. Vlasov, G. Kozina, T. Kostinskaya, L. Kurbatov, and A. Uvarov, "Continuously tunable electron-beam-pumped semiconductor laser," Quantum Electronics, vol. 7, pp. 1168-1169, 1977.

[56] S. Demidov, E. Bibikov, A. Vlasov, G. Kozina, and G. Saigina, "Resonators with enlarged-area exit mirrors for electron-beam lasers," Soviet Journal of Quantum Electronics, vol. 4, p. 607, 1974.

[57] O. V. Bogdankevich, N. Vorob'ev, M. Zverev, S. Kopyt, E. Krasavina, I. Kryukova, et al., "Pulsed multielement semiconductor laser with an unstable resonator," Quantum Electronics, vol. 15, pp. 1002-1003, 1985.

[58] F. Tong, R. Osgood, A. Sanchez, and V. Daneu, "Electron-beam-pumped twodimensional semiconductor laser array with tilted mirror resonator," Applied physics letters, vol. 52, pp. 1303-1305, 1988.

[59] O. V. Bogdankevich, M. Zverev, A. Pechenov, and B. I. Vasil'ev, "Multielement semiconductor laser of the" emitting mirror" type," Kvantovaya Elektronika, pp. 95-96, 1971.

[60] S. Demidov, G. Kozina, L. Kurbatov, A. Myasnikov, and V. Rudnevski1̌, "Compact infrared laser source based on a field-emission electrontube," Soviet Journal of Quantum Electronics, vol. 8, p. 1159, 1978.

[61] O. V. Bogdankevich, M. Zverev, N. Kostin, S. Kopyt, E. Krasavina, I. Kryukova, et al., "Uncooled pulsed cadmium sulfide and gallium arsenide lasers pumped longitudinally by an electron beam," Quantum Electronics, vol. 15, pp. 1000-1002, 1985.

[62] O. Bogdankevich, M. Zverev, A. Mestvirishvili, A. Nasibov, A. Pechenov, A. Svinenkov, et al., "Electron-beam-pumped high-power semiconductor laser," Quantum Electronics, vol. 1, pp. 184-185, 1971.

[63] A. Braginskaya, V. Kozlovskiı̌, G. Kolchina, B. M. Lavrushin, A. S. Nasibov, and P. Reznikov, "Scanned and cw GaSb lasers with longitudinal electron beam pumping," Quantum Electronics, vol. 15, pp. 551-553, 1985.

[64] O. V. Bogdankevich, B. Bryunetkin, S. Darznek, M. Zverev, and V. Ushakhin, "Two-dimensional variable-gap structures in longitudinally pumped semiconductor lasers," Quantum Electronics, vol. 8, pp. 1148-1150, 1978. 
[65] J. R. Packard, W. C. Tait, and G. H. Dierssen, "Two-Dimensionally Scannable Electron-Beam-Pumped Laser," Applied Physics Letters, vol. 19, pp. 338-340, 1971.

[66] G. Kotovshchikov, V. Kuklev, N. Lantsov, G. Meerovich, A. Negodov, and V. Ulasyuk, "Sealed scanned electron-beam-excited semiconductor laser," Quantum Electronics, vol. 4, pp. 242-243, 1974.

[67] O. V. Bogdankevich, M. Zverev, and A. Pechenov, "Divergence of the output radiation of electron-beam-pumped" radiating mirror" lasers," Kvantovaya Elektronika, pp. 110-111, 1972.

[68] S. Darznek, M. Zverev, and V. Ushakhin, "Investigation of a multielement electronbeam-pumped semiconductor laser with an external mirror," Quantum Electronics, vol. 4, pp. 1272-1274, 1975.

[69] N. Basov, O. Bogdankevich, and A. Grasyuk, "9B4-Semiconductor lasers with radiating mirrors," Quantum Electronics, IEEE Journal of, vol. 2, pp. 594-597, 1966.

[70] O. Bogdankevich, S. Darznek, A. Pechenov, B. Vasiliev, and M. Zverev, "Semiconductor electron-beam-pumped lasers of the radiating mirror type," Quantum Electronics, IEEE Journal of, vol. 9, pp. 342-347, 1973.

[71] N. Basov, O. Bogdankevich, and A. Devyatkov, "Exciting of a semiconductor quantum generator with a fast electron beam\#," Начало лазерной эры в СССР, p. $76,1964$.

[72] N. Basov, O. Bogdankevich, A. Nasibov, V. I. Kozlovsky, V. Papusha, and A. Pechenov, "Formation of a television image on a large screen with the aid of a laser electron-beam tube," Quantum Electronics, vol. 4, pp. 1408-1408, 1975.

[73] I. Bogdankevich, O. V. Bogdankevich, S. Darznek, M. Zverev, L. Tumanova, and V. Ushakhin, "Lasing threshold and divergence of radiation emitted by semiconductor lasers pumped longitudinally by an electron beam," Quantum Electronics, vol. 15, pp. 553-555, 1985.

[74] O. Bogdankevich, S. Darznek, M. Zverev, and V. Ushakhin, "Influence of amplified spontaneous radiation on parameters of a longitudinally pumped laser," Quantum Electronics, vol. 5, pp. 953-956, 1975.

[75] O. Bogdankevich, V. Djukov, S. Beljaev, S. Gavrikov, and L. Nevzorova, "Scanning laser microscope," Quantum Electronics, IEEE Journal of, vol. 16, pp. 129-131, 1980.

[76] O. Bogdankevich, S. Korolev, A. Nasedkin, I. Olikhov, and D. Petrov, "Pumping of a semiconductor laser by an electron beam modulated at a microwave frequency," Quantum Electronics, vol. 1, pp. 386-388, 1972. 
[77] O. Bogdankevich and V. Ulasyuk, "Influence of the excitation inhomogeneity on the threshold of electron-beam-pumped lasers," Soviet Journal of Quantum Electronics, vol. 4, p. 198, 1974.

[78] M. M. Zverev, N. A. Gamov, D. V. Peregoudov, V. B. Studionov, E. V. Zdanova, I. V. Sedova, et al., "An efficient electron-beam-pumped semiconductor laser for the green spectral range based on II-VI multilayer nanostructures," Semiconductors, vol. 42, pp. 1440-1444, 2008/12/01 2008.

[79] E. N. Donskoi, E. V. Zhdanova, A. N. Zalyalov, M. M. Zverev, S. V. Ivanov, D. V. Peregudov, et al., "Excitation density distribution in electron-beam-pumped ZnSe semiconductor lasers," Quantum Electronics, vol. 38, pp. 1097-1100, 2008.

[80] M. M. Zverev, S. V. Sorokin, I. V. Sedova, S. V. Ivanov, P. S. Kop'ev, and D. V. Peregoudov, "High-efficiency electron-beam pumped green semiconductor lasers based on multiple CdSe quantum disk sheets," physica status solidi (c), vol. 2, pp. 923-926, 2005.

[81] M. M. Zverev, S. V. Sorokin, I. V. Sedova, D. V. Peregoudov, S. V. Ivanov, and P. S. Kopev, "ZnSe-Based Room Temperature Low-Threshold Electron-Beam Pumped Semiconductor Laser," physica status solidi (b), vol. 229, pp. 1025-1028, 2002.

[82] S. V. Gronin, S. V. Sorokin, I. V. Sedova, S. V. Ivanov, E. V. Zdanova, and M. M. Zverev, "ZnSe-based laser structures for electron-beam pumping with graded index waveguide," physica status solidi (c), vol. 7, pp. 1694-1696, 2010.

[83] C. E. Hurwitz and R. J. Keyes, "Electron-beam-pumped GaAs laser," Applied Physics Letters, vol. 5, pp. 139-141, 1964.

[84] I. Kryukova, V. Leskovich, and E. Matveenko, "Mechanisms of laser action in epitaxial InAs subjected to electron beam excitation," Soviet Journal of Quantum Electronics, vol. 9, p. 823, 1979.

[85] N. Holonyak Jr, J. C. Campbell, M. H. Lee, J. T. Verdeyen, W. L. Johnson, M. G. Craford, et al., "Pumping of GaAs $1-\mathrm{x} \mathrm{P}_{\mathrm{x}}: \mathrm{N}$ (at $77 \mathrm{~K}$, for $\left.\mathrm{x} \leq 0.53\right)$ by an electron beam from a gas plasma," Journal of Applied Physics, vol. 44, pp. 5517-5521, 1973.

[86] J. K. Shultis and R. E. Faw, Fundamentals of Nuclear Science and Engineering: Taylor \& Francis, 2002.

[87] M. B. Chadwick, M. Herman, P. Obložinský, M. E. Dunn, Y. Danon, A. C. Kahler, et al., "ENDF/B-VII.1 Nuclear Data for Science and Technology: Cross Sections, Covariances, Fission Product Yields and Decay Data," Nuclear Data Sheets, vol. 112, pp. 2887-2996, 2011/12/01/ 2011.

[88] J. F. Ziegler, M. D. Ziegler, and J. P. Biersack, "SRIM - The stopping and range of ions in matter (2010)," Nuclear Instruments and Methods in Physics Research 
Section B: Beam Interactions with Materials and Atoms, vol. 268, pp. 1818-1823, 2010/06/01/ 2010.

[89] NASA, "Mars Science Laboratory Landing Press Kit," ed, 2011.

[90] B. D. Weaver, D. McMorrow, and L. M. Cohn, "Radiation effects in III-V semiconductor electronics," International Journal of High Speed Electronics and Systems, vol. 13, pp. 293-326, 2003.

[91] M. Prelas, M. Boraas, F. De La Torre Aguilar, J. D. Seelig, M. T. Tchouaso, and D. Wisniewski, Nuclear Batteries and Radioisotopes: Springer International Publishing, 2016.

[92] I. Waki, H. Fujioka, M. Oshima, H. Miki, and A. Fukizawa, "Low-temperature activation of Mg-doped GaN using Ni films," Applied Physics Letters, vol. 78, pp. 2899-2901, 2001.

[93] O. Bogdankevich, V. Goncharov, B. Lavrushin, V. Letokhov, and A. Suchkov, "Effects of excitation inhomogeneity in semiconductor lasers pumped by an electron beam," SOVIET PHYSICS-SEMICONDUCTORS, vol. 1, pp. 4-9, 1967.

[94] O. Bogdankevich, V. Letokhov, and A. Suchkov, "Theory of the effects of inhomogeneous excitation of semiconductor lasers using electron beam pumping," FIZIKA I TEKHNIKA POLUPROVODNIKOV, vol. 3, pp. 665-670, 1969.

[95] A. F. Suchkov, "Effect of Inhomogeneities on the Operation Regime of Solid-state Lasers," Journal of Experimental and Theoretical Physics, vol. 22, pp. 1026-1031, 1966.

[96] W. D. J. Jr., "Characteristics of Optically Pumped Platelet Lasers of ZnO, CdS, CdSe, and CdS0.6Se0.4 Between $300^{\circ}$ and $80^{\circ} \mathrm{K}$," Journal of Applied Physics, vol. 42, pp. 2731-2740, 1971.

[97] J. T. Verdeyen, Laser Electronics: Prentice Hall, 1995.

[98] K. J. Kuhn, Laser Engineering: Prentice Hall, 1998.

[99] B. E. A. Saleh and M. C. Teich, Fundamentals of Photonics: Wiley, 2007.

[100] M. Prelas, "Nuclear-Pumped Lasers," in Nuclear-Pumped Lasers, ed Cham: Springer International Publishing, 2016, pp. 131-228.

[101] S. T. Revankar and T. E. Adams, "Advances in betavoltaic power sources," $J$. Energy Power Sources, vol. 1, pp. 321-329, 2014.

[102] J. R. Taylor, Introduction To Error Analysis: The Study of Uncertainties in Physical Measurements: University Science Books, 1997. 
[103] M. Kubíček and V. Hlaváček, Numerical solution of nonlinear boundary value problems with applications: Prentice-Hall, 1983. 


\section{Vita}

Denis Wisniewski was born in Kansas City, Missouri to George \& Martha Wisniewski. Denis was homeschooled for most of his childhood until he began attending the Kansas City Metropolitan Community College system at the age of 16 . He received his BS in physics from the University of Missouri in 2011, MS in nuclear engineering from the University of Missouri in 2012. He has authored on 7 journal papers and one book. 